\title{
Experimental investigation on vibration characteristics of the medium-low-speed maglev vehicle-turnout coupled system
}

\author{
Miao $\mathrm{Li}^{1} \cdot$ Dinggang Gao ${ }^{1,2} \cdot \mathrm{Tie}^{\mathrm{Li}^{1,3}} \cdot$ Shihui $\mathrm{Luo}^{1} \cdot$ Weihua $\mathrm{Ma}^{1}$ • \\ Xiaohao Chen ${ }^{1}$
}

Received: 24 August 2021/Revised: 18 November 2021/Accepted: 18 November 2021/Published online: 30 December 2021

(C) The Author(s) 2021

\begin{abstract}
The steel turnout is one of the key components in the medium-low-speed maglev line system. However, the vehicle under active control is prone to vehicle-turnout coupled vibration, and thus, it is necessary to identify the vibration characteristics of this coupled system through field tests. To this end, dynamic performance tests were conducted on a vehicle-turnout coupled system in a medium-low-speed maglev test line. Firstly, the dynamic response data of the coupled system under various operating conditions were obtained. Then, the natural vibration characteristics of the turnout were analysed using the free attenuation method and the finite element method, indicating a good agreement between the simulation results and the measured results; the acceleration response characteristics of the coupled system were analysed in detail, and the ride quality of the vehicle was assessed by Sperling index. Finally, the frequency distribution characteristics of the coupled system were discussed. All these test results could provide references for model validation and optimized design of medium-low-speed maglev transport systems.
\end{abstract}

Keywords Medium-low-speed maglev · Vehicle-turnout coupled system · Field test · Vibration characteristics · Ride quality

Dinggang Gao

12138@tongji.edu.cn

1 State Key Laboratory of Traction Power, Southwest Jiaotong University, Chengdu 610031, China

2 Maglev Transportation Engineering R\&D Center, Tongji University, Shanghai 201804, China

3 City Rail Technical Development Department, CRRC Dalian Locomotive and Rolling Stock Co., Ltd, Dalian 116022, China

\section{Introduction}

In order to alleviate traffic congestion, urban rail transit has been promoted rapidly in recent years, greatly facilitating the travelling of passengers [1-3]. Medium-low-speed maglev, as one of the new urban rail transit systems, has considerable development potentials due to its excellent features such as low-noise operation, route selection flexibility, zero derailment risk and short construction cycle, and thus has been put into commercial operation in Japan, Korea and China [4-6]. All these commercial lines use normal conducting magnetic levitation; however, practice has shown that vehicle/guideway dynamic interaction with active control is problematic, and the vehicle is prone to levitation instability when standing still above the guideway as well as vehicle-guideway coupled vibration $[1,7-9]$, posing a challenge for the further development of medium-low-speed maglev transport.

The problem of the dynamic interaction between vehicle and guideway has been extensively studied in rail transport dominated by wheel/rail system [10-14]. In the past decade or so, many scholars around the world have carried out a series of studies and made significant contributions to vehicle-guideway coupled vibration in normal conducting magnetic levitation: Yaghoubi and Ziari [15] discussed the design methods and criteria for guideway structure systematically; Han et al. [16] investigated the effect of guideway vibration characteristics on the dynamic performance of the vehicle by means of numerical simulations and field tests; Kim et al. [17] developed a detailed vehicle-guideway dynamics model to study its vibration characteristics when standing still and moving at low speeds; Lee et al. [18] investigated the effects of parameters such as vehicle speed, irregularity, deflection/span ratio, bridge 
span and bridge damping ratio on the dynamics of medium-low-speed maglev vehicles and on flexible guideway bridges; Kwon et al. [19], Wang et al. [20] and Yau et al. $[21,22]$ explored the dynamic effects of gusty wind, ground settlement and earthquakes on vehicle and guideway; Han et al. [23] analysed the characteristics of vehicleguideway dynamic interaction and proposed a limit value for the deflection/span ratio of the bridge; Chen et al. [24] put forward a method to identify the linear and nonlinear stability of the levitation systems and plotted the stability domain of the system in the parameter space; Wang et al. $[25,26]$ also investigated the effect of guideway structure on the dynamics of the coupled system; Han et al. [27] evaluated the levitation stability of the vehicle running on turnouts by numerical simulation. In terms of line testing, $\mathrm{Li}$ et al. [28] and $\mathrm{Li}$ et al. [29] conducted experimental studies on the dynamic performance between vehicle and concrete guideway on China's first commercial mediumlow-speed maglev line (Changsha Maglev Express) and a certain medium-low-speed test line, respectively. In terms of levitation control and its theoretical research, Sun et al. [30] proposed a new approach using Internet of Things and adaptive fuzzy control; Chen et al. [31] focused on the parameter identification of nonlinear dynamic model of a maglev system and the design of radial basis function (RBF) network adaptive control algorithm and verified the reliability of identification results and control algorithm; Kong et al. [32] proposed a Kalman filter-based sliding mode control method that can reduce the levitation gap fluctuation and improve the vertical dynamic response of the vehicle; Li and Zhang [33] analysed the influence of the levitation control algorithm and main parameters on the levitation stability and proposed an expression for the relationship between stability and system parameters; Wang et al. [34] and Zhang et al. [35] studied the stability and dynamics behaviour of levitation systems with delayed position and speed feedback.

Despite all these progresses, existing studies have paid little attention to the dynamic response of the vehicleturnout coupled system when the vehicle is running on a lightweight turnout, resulting in a lack of test data in this field. The steel turnout, as a device that helps change the running route of the train, however, is one of the most critical structures in the medium-low-speed maglev system, and one of the key factors affecting the normal operation of the vehicle as its design involves so many aspects such as structural strength, mechanical movement, coupled vibration and particularly the vehicle-turnout dynamic interaction. Therefore, to identify the dynamic response characteristics of the vehicle-turnout coupled system under different operating conditions, it is of vital importance to carry out corresponding field tests on the coupled system and to analyse its vibration characteristics in detail based on the test data obtained.

Thus, field tests are conducted on dynamic performance of the vehicle and turnout on a medium-low-speed maglev test line, and accordingly, the remainder of this paper is arranged as follows: Sect. 2 gives a brief introduction to the structural characteristics of the vehicle-turnout coupled system and illustrates the test scheme; Sect. 3 first analyses the natural vibration characteristics of the turnout subsystem, then examines the dynamic response characteristics of the coupled system under different operating conditions in both the time and frequency domains, and subsequently, evaluates the ride quality of vehicle by Sperling index; finally, the main conclusions and further research directions are given in Sect. 4.

\section{Field tests of the vehicle-turnout coupled system}

The selected medium-low-speed maglev test line has a total length of approximately $1.73 \mathrm{~km}$, a minimum horizontal curve radius of $50 \mathrm{~m}$, a transition curve in the form of clothoid, a maximum cross-slope angle of $6^{\circ}$, a crossslope torsion rate of $0.12 \% \mathrm{~m}$ within the transition curve, a minimum vertical curve radius of $1500 \mathrm{~m}$, a maximum slope of $70 \%$ and a track gauge of $1900 \mathrm{~mm}$. The main and auxiliary lines are connected by the turnout, as shown in Fig. 1.

\subsection{Test vehicle}

The running mechanism of the test vehicle consists of five levitation frames, all of which are powered, retaining not only the function of levitation, guiding, traction and braking of the train, but also the ability to decouple the movements in order to adapt to various twists and irregularities of the lines. Figure 2 shows a schematic 3D model

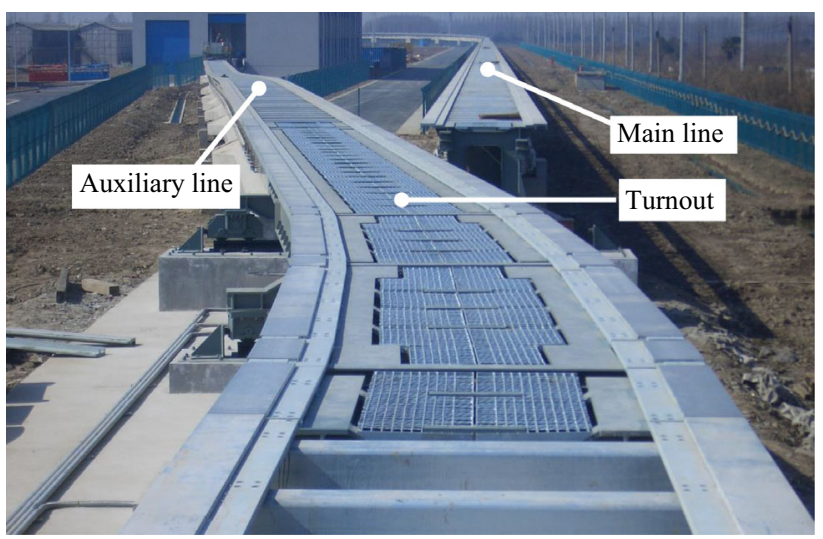

Fig. 1 Test line 


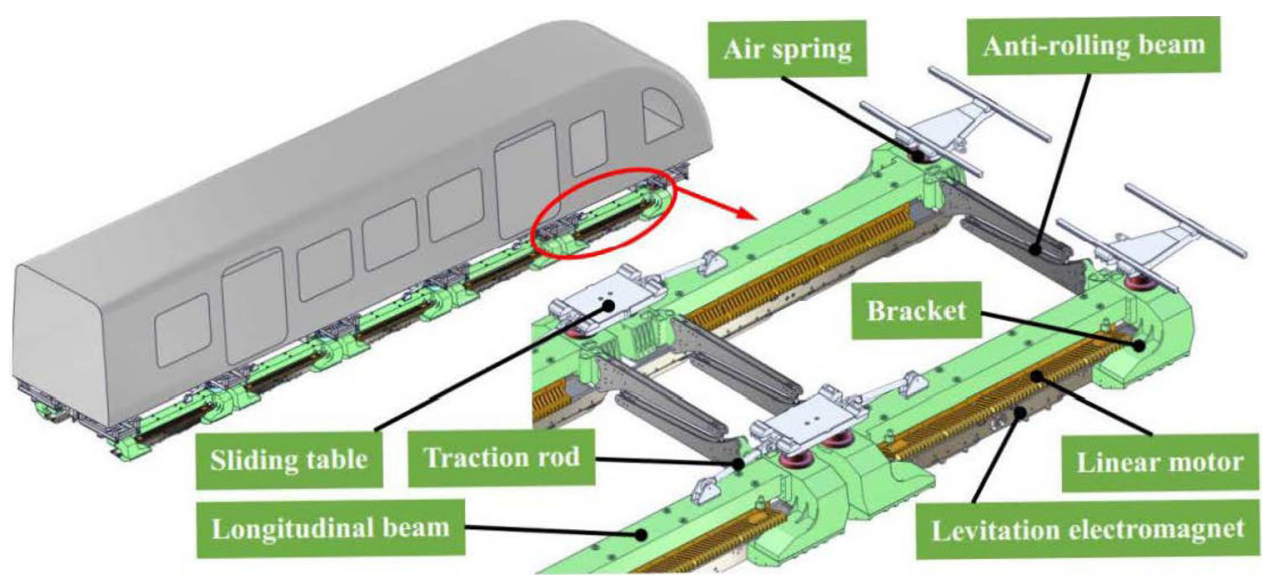

Fig. 2 Test vehicle and key structural components of the levitation frame

Table 1 Main technical parameters of the test vehicle

\begin{tabular}{lll}
\hline Name & Value & Unit \\
\hline Mass of the test vehicle & 21 & $\mathrm{t}$ \\
$\begin{array}{l}\text { Mass of levitation frame } \\
\text { Longitudinal centre-to-centre spacing between }\end{array}$ & 2880 & $\mathrm{t}$ \\
$\quad$ adjacent levitation frames & 1978 & $\mathrm{~mm}$ \\
$\begin{array}{l}\text { Lateral span of air spring } \\
\text { Length of levitation electromagnet }\end{array}$ & 2800 & $\mathrm{~mm}$ \\
$\begin{array}{l}\text { Lateral centre-to-centre spacing between } \\
\text { electromagnets }\end{array}$ & 1900 & $\mathrm{~mm}$ \\
$\begin{array}{l}\text { Vertical stiffness of air spring } \\
\text { Lateral stiffness of air spring }\end{array}$ & $8 \times 10^{4}$ & $\mathrm{~N} / \mathrm{m}$ \\
\hline
\end{tabular}

of the test vehicle and highlights the key structural components of its levitation frames, which have an overall " $\square$ "-shaped layout and consist mainly of levitation electromagnets, linear motors, longitudinal beams, brackets, air springs, anti-rolling beams, support wheel mounting base, etc. Among these components, longitudinal beams, brackets, bracket connectors and anti-rolling beams constitute the bogie of the levitation frame, upon which the levitation electromagnets, linear motors and the air spring suspension system are mounted, making it the core structural component of the running mechanism. The main technical parameters of the test vehicle are listed in Table 1.

\subsection{Turnout of steel structure}

The turnout of the medium-low-speed maglev system is a single joint turnout with three segments. The change of the running route from the main line to auxiliary line is achieved by the motor-driven long-span girder (LSG), and the circular curve is fitted by straight lines. When the turnout is in the curved position (the vehicle switching to the auxiliary line), the LSG, short-span girder \#1(SSG \#1) and short-span girder \#2 (SSG \#2) form polygonal lines to fit the circular curve, with no transition curve at both ends; when the turnout is in the straight direction (the vehicle moving straight ahead long the main line), the line of the turnout is ideally straight. The turnout adopted in our test is a left-hand steel structure, which consists of two simply supported SSGs (not motor-driven) and a continuous girder with vertical support in the middle of the span (namely the motor-driven LSG mentioned above), as shown in Fig. 3. The LSG, SSGs and support girders at the two ends are all made of steel. The LSG is approximately $19,000 \mathrm{~mm}$ and the two SSGs are $4500 \mathrm{~mm}$ each. The cross section of the girders is a double-web structure with a web thickness of $16 \mathrm{~mm}$, an upper flange width of $315 \mathrm{~mm}$, a lower flange width of $200 \mathrm{~mm}$, an upper and lower flange plate thickness of $24 \mathrm{~mm}$ and a spacing of $1200 \mathrm{~mm}$ between the left and right webs.

\subsection{Description of the test scheme}

The test conditions are described as follows: the vehicle passed the turnout along the main line and auxiliary line with no load, running at speeds of 10 to $60 \mathrm{~km} / \mathrm{h}$ (at $10 \mathrm{~km} /$ $\mathrm{h}$ intervals) and 10 to $15 \mathrm{~km} / \mathrm{h}$ (at $5 \mathrm{~km} / \mathrm{h}$ intervals), respectively; besides, the test condition of the vehicle standing still above the LSG was also examined. To explore the dynamics behaviour of the vehicle-turnout coupled system, some measurement points were arranged. For the vehicle subsystem, two measurement points were placed at each position, such as the vehicle floor and the bracket connectors of the levitation module on the right side of the levitation frame \#2, as shown in Fig. 4a, collecting data of levitation gap from two control points on the right levitation module of levitation frame \#2 (namely levitation points \#5 and \#7) together with levitation gap 


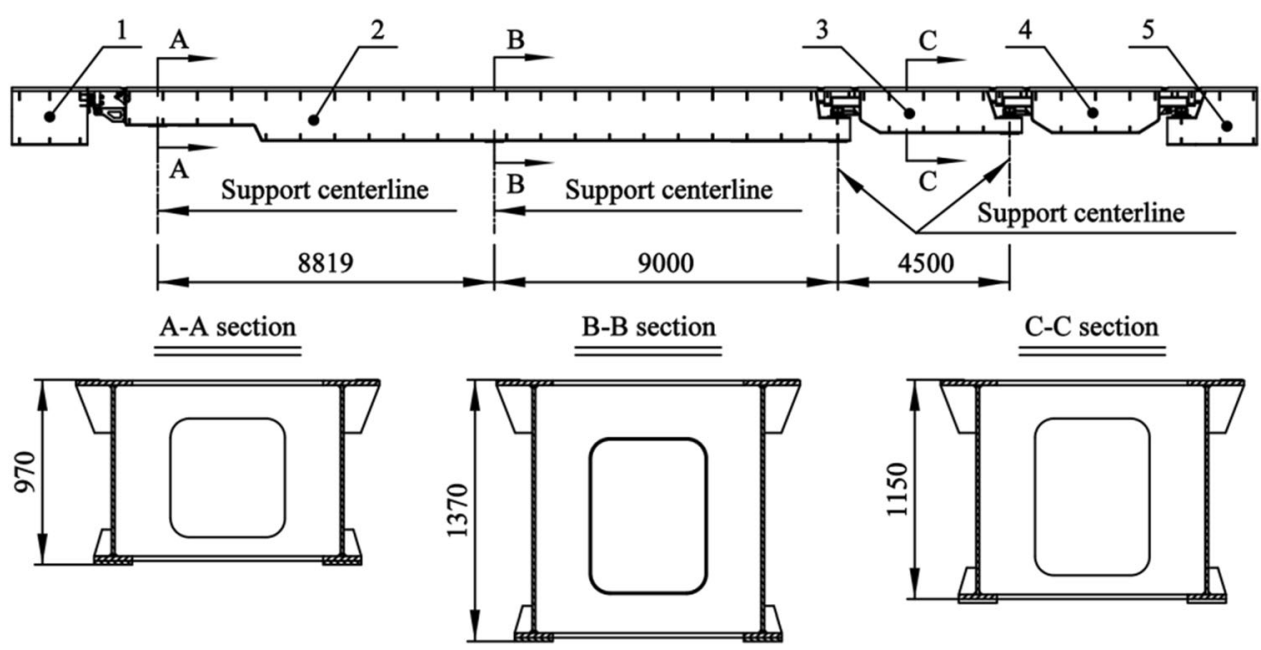

1: Support girder at the free end; 2: Long-span girder (LSG); 3: Short-span girder \#1 (SSG \#1); 4: Short-span girder \#2 (SSG \#2); 5: Support girder at the fixed end

Fig. 3 Schematic diagram of the turnout structure (unit: $\mathrm{mm}$ )

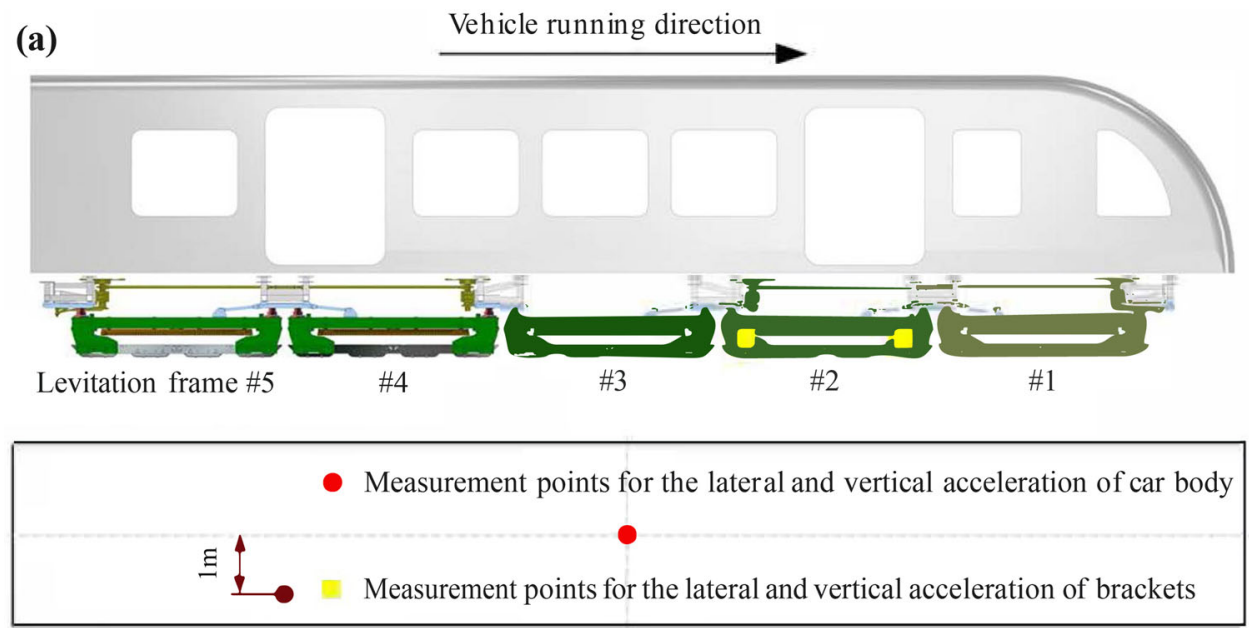

(b)

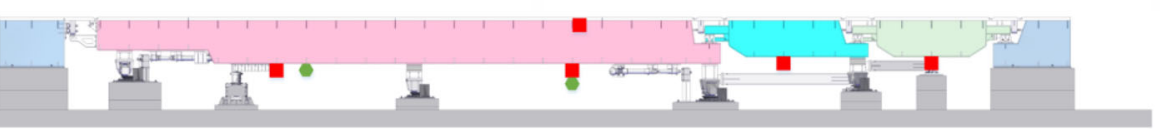

Front view

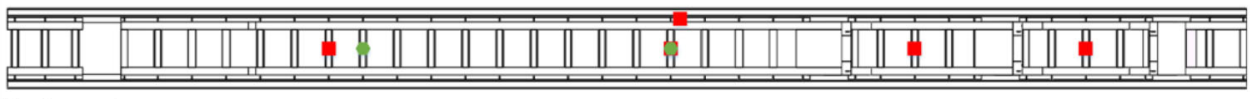

Bottom view

- Measurement points for the lateral and vertical acceleration

- Measurement points for dynamic displacement

Fig. 4 Measurement points of vehicle-turnout coupled system: a measurement points of vehicle subsystem; b measurement points of turnout subsystem

sensor. As for the turnout subsystem, a total of seven measurement points were placed at the LSG, SSG \#1, SSG \#2 and so on, as shown in Fig. 4b. The information on the measurement points is summarized in Table 2 .
In terms of test equipment, piezoelectric acceleration sensors were selected to collect the vibration acceleration signals of the vehicle-turnout coupled system, and laser displacement sensors were used to collect the vertical 
Table 2 Measurement points of the vehicle-turnout coupled system

\begin{tabular}{|c|c|c|c|c|}
\hline \multicolumn{2}{|l|}{ Measurement position } & \multirow{2}{*}{$\begin{array}{l}\text { Measurement point } \\
\text { Centre }\end{array}$} & \multirow{2}{*}{$\frac{\text { Direction }}{\text { Lateral/vertical }}$} & \multirow{2}{*}{$\begin{array}{l}\text { Type } \\
\text { Acceleration }\end{array}$} \\
\hline Vehicle subsystem & Car body & & & \\
\hline & & Right side of the rear end & & \\
\hline & Right module of the & Front-end bracket & & \\
\hline & levitation frame \#2 & Rear-end bracket & & \\
\hline \multirow[t]{7}{*}{ Turnout subsystem } & LSG & Mid-span of the 1st span & Lateral/vertical & Acceleration \\
\hline & & & Vertical & Dynamic displacement \\
\hline & & Mid-span of the 2 nd span & Lateral/vertical & Acceleration \\
\hline & & & Vertical & Dynamic displacement \\
\hline & & Upper flange & Lateral/vertical & Acceleration \\
\hline & SSG \#1 & Mid-span & Lateral/vertical & Acceleration \\
\hline & SSG \#2 & Mid-span & & \\
\hline
\end{tabular}

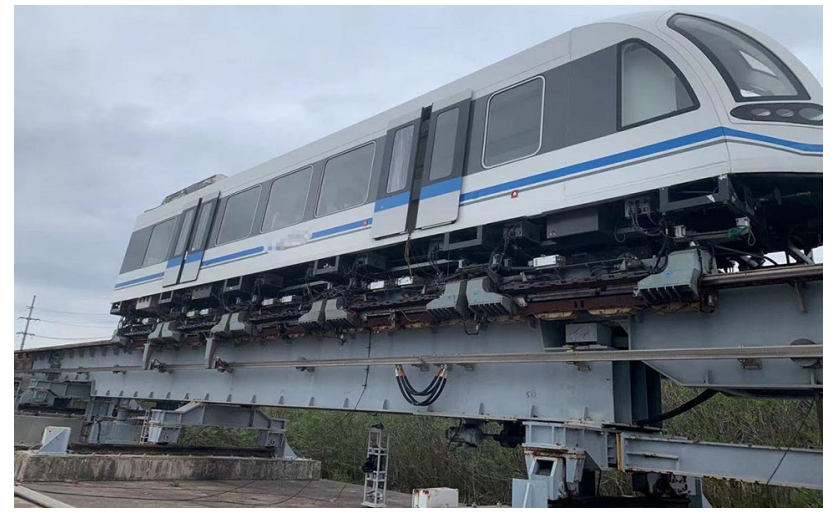

Fig. 5 Field test scene

dynamic displacement signals of the LSG. The dynamic response signals of the vehicle and turnout subsystems were collected by WaveBook-WBK18 and SIRIUS dynamic data systems, which had a sampling frequency of 1000 and $5000 \mathrm{~Hz}$, respectively. Field test scene is shown in Fig. 5.

\section{Results and discussion}

\subsection{Natural vibration characteristics of the turnout}

\subsubsection{Measured data}

Natural frequency is one of the most important factors influencing the vibration characteristics of a structure, and the turnout subsystem is no exception. Generally, natural frequency can be identified by ambient excitation or by the natural vibration attenuation curve of the residual vibration waveform after the vehicle has passed. Figure 6 shows the dynamic displacement and time history of vibration acceleration of LSG in the vertical direction when the test vehicle passes the turnout along the main line. According to Fig. 6, the vertical dynamic displacement and acceleration of the LSG go through three phases: A, B and C, which correspond to the states before the vehicle enters the LSG, when the vehicle passes it through and when the vehicle exits, respectively. By performing a fast Fourier transform on the vibration acceleration data in phase $\mathrm{C}$ at each measurement point on the turnout, the natural vibration characteristics of the turnout subsystem are obtained. Figure 7 indicates the results of multiple tests of the natural vibration spectrum of the LSG and SSGs in the vertical and lateral directions. It is clear that the spectrogram under the three tests retain consistency, indicating that the test results are reliable. Both the girders have low-frequency vibration components below $5 \mathrm{~Hz}$, which may be caused by the special structure of the turnout, such as the existence of mechanical gaps or the possible presence of rigid body frequencies in the turnout subsystem, as both the LSG and SSGs are supported by trolleys, with support stiffness weaker than concrete girders commonly used on the main line. The frequency distribution of the both girders is wide, indicating that the turnout subsystem has a rich variety of vibration modes.

As shown in Fig. 7a, c, in the lateral and vertical directions, the LSG has similar frequency components at several frequencies (e.g. 19.2, 25.2, 28.6, 49.6, 64.6, 84.8 and $91.2 \mathrm{~Hz}$ ), indicating that it is rich in torsional vibration modes; at $14.2 \mathrm{~Hz}$, the lateral bending natural vibration frequency of the LSG dominates, accompanied by torsional vibration; around $27.0 \mathrm{~Hz}$, the vertical bending natural vibration frequency of LSG dominates. According to Fig. 7b, d, SSG has more dominant high-frequency vibration components than LSG due to the shorter support span; within several frequency ranges, SSG also has the same local peak frequencies in the vertical and lateral directions (e.g. 25.2, 28.6, 58.6, 60.8, 66.8 and $88.4 \mathrm{~Hz}$ ), indicating that it also has a rich variety of vibration modes. 
(a)

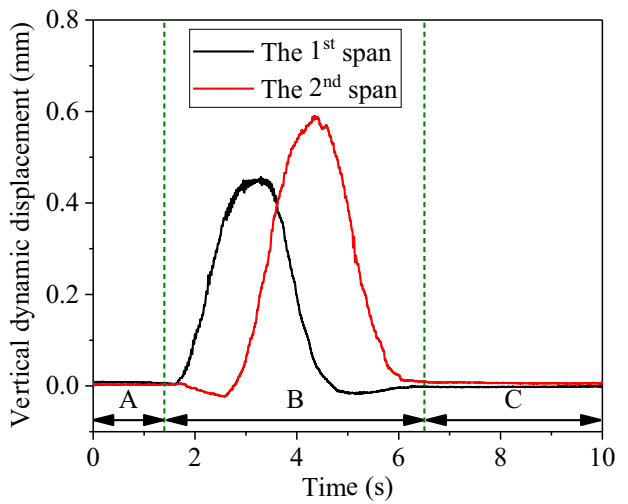

(b)

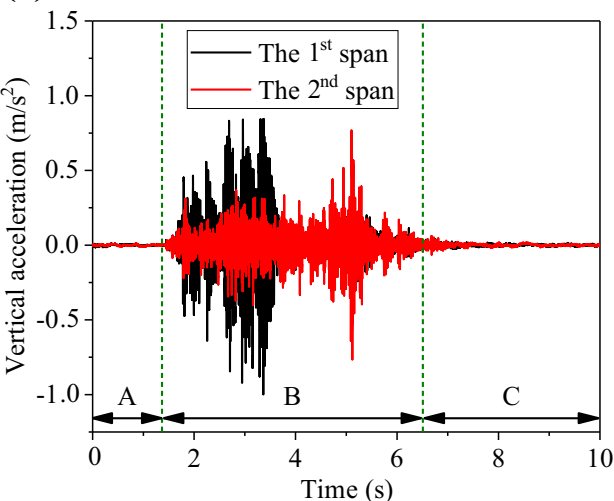

Fig. 6 Dynamic response time history of LSG in the vertical direction when the vehicle passes along the main line: a dynamic displacement; b acceleration

(a)

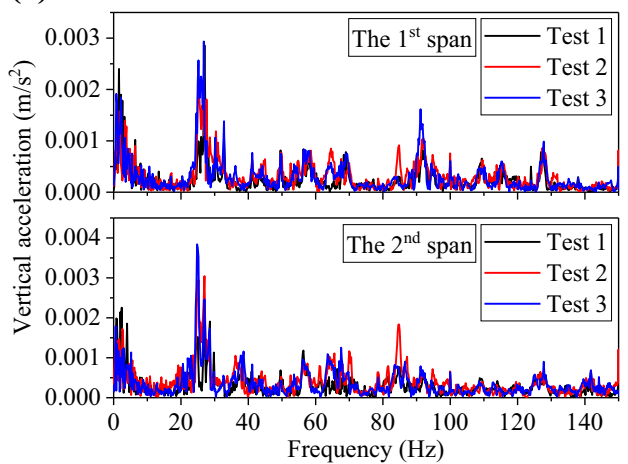

(c)

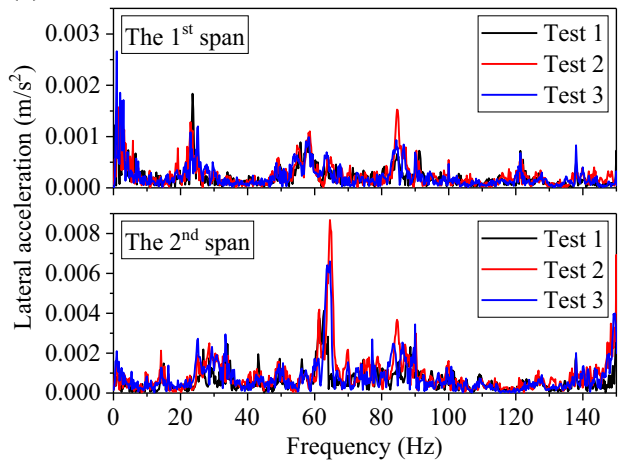

(b)

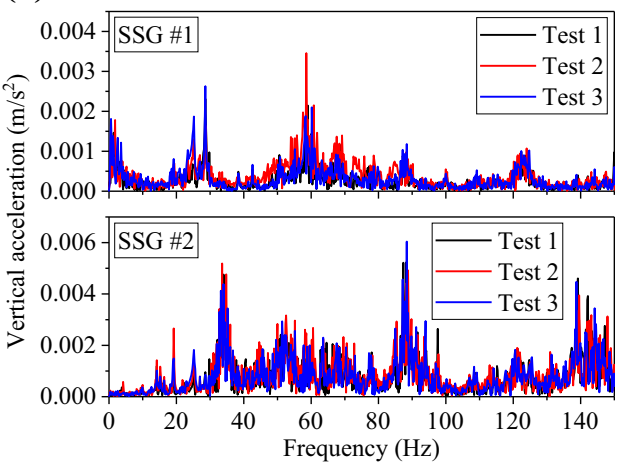

(d)

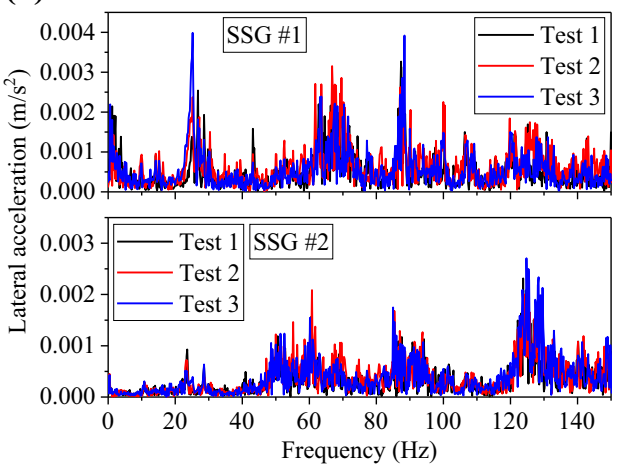

Fig. 7 Natural vibration spectra: a LSG in the vertical direction; b SSG in the vertical direction; $\mathbf{c}$ LSG in the lateral direction; d SSG in the lateral direction

\subsubsection{Numerical simulation}

To better understand the natural vibration characteristics of the turnout, a finite element model was built using HyperMesh. The model contains LSG, SSGs and support girders, as shown in Fig. 8. Solid185 in ANSYS was used for F-rail, shell63 was used for the rest of the structures, and LSG was constrained in a way referenced in the literature [36]. As turnout girders are significantly weaker than ordinary concrete bridges in terms of actual restraint strength [37], elastic restraint was used to simulate the trolley supporting the turnout. With measured results considered, the order of magnitude of the support stiffness was taken at $1 \times 10^{8} \mathrm{~N} / \mathrm{m}$. Table 3 shows the typical vibration for the turnout model. Considering the fact that the LSG in the test line of CRRC Zhuzhou Electric 


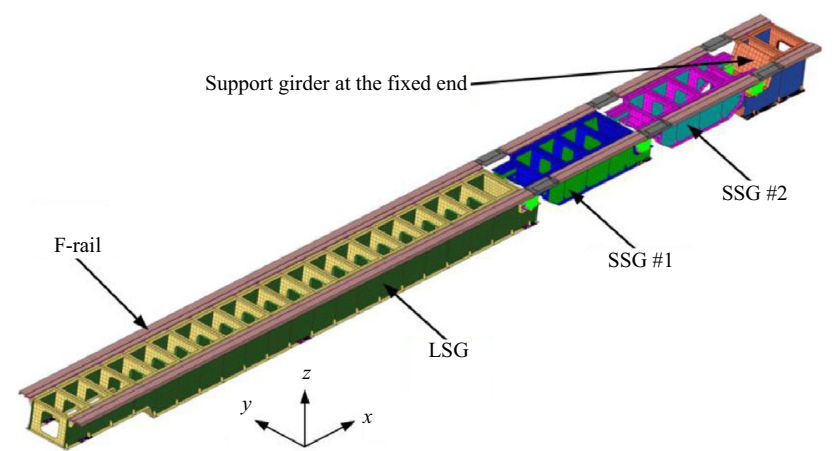

Fig. 8 3D finite element model of the turnout

Locomotive Co., Ltd. was designed without support trolley in the middle [38], we also carried out modal calculations for the turnout scheme with two trolleys supporting the LSG by making the force element supporting the middle position of LSG ineffective and keeping the rest of the parameters. The results obtained from the numerical calculations are summarized in Table 4.

The modal results show that the turnout is indeed rich in modal information, with few independent vibration patterns in a single direction but mostly complex modal vibration patterns. When LSG is supported by a trolley in the middle, the lateral bending and vertical bending natural frequencies of the turnout are 7.64 and $25.62 \mathrm{~Hz}$, respectively, while the corresponding values are 7.14 and $10.08 \mathrm{~Hz}$ when there is no trolley supporting in the middle. It is evident that the support stiffness of the turnout is significantly increased with trolley supporting in the middle of LSG, which has a greater effect on the vertical bending natural frequency of the turnout than in the lateral direction, because the middle trolley mainly restrains the vertical motion of LSG; besides, the frequencies 14.22, $19.31,22.65,26.08,29.18,47.32,66.71 \mathrm{~Hz}$, etc. in Table 3 are in good agreement with the measured results in Fig. 7. These frequencies correspond mainly to lateral bending and torsional vibration patterns. As for vertical bending vibration pattern, frequencies of the first two orders obtained by numerical calculations are 25.62 and $30.26 \mathrm{~Hz}$, respectively, which also agree well with the measured frequencies around $27 \mathrm{~Hz}$.

It should be further noted that both Changsha Maglev Express and Qingyuan Maglev Tourism Special Line in Guangdong use similar LSG, with the vertical bending natural frequencies in the range of $15-19 \mathrm{~Hz}$, which differs significantly from the measured and simulated results in this paper, probably because of the greater vertical support stiffness provided by the middle trolley in the test line (as reflected in Fig. 6a). In Ref. [39], a dominant frequency of $23.97 \mathrm{~Hz}$ for vertical vibration of LSG in Changsha Maglev Express under ambient excitation was measured,
Table 3 Natural vibration modes of the turnout with 3 trolleys supporting LSG

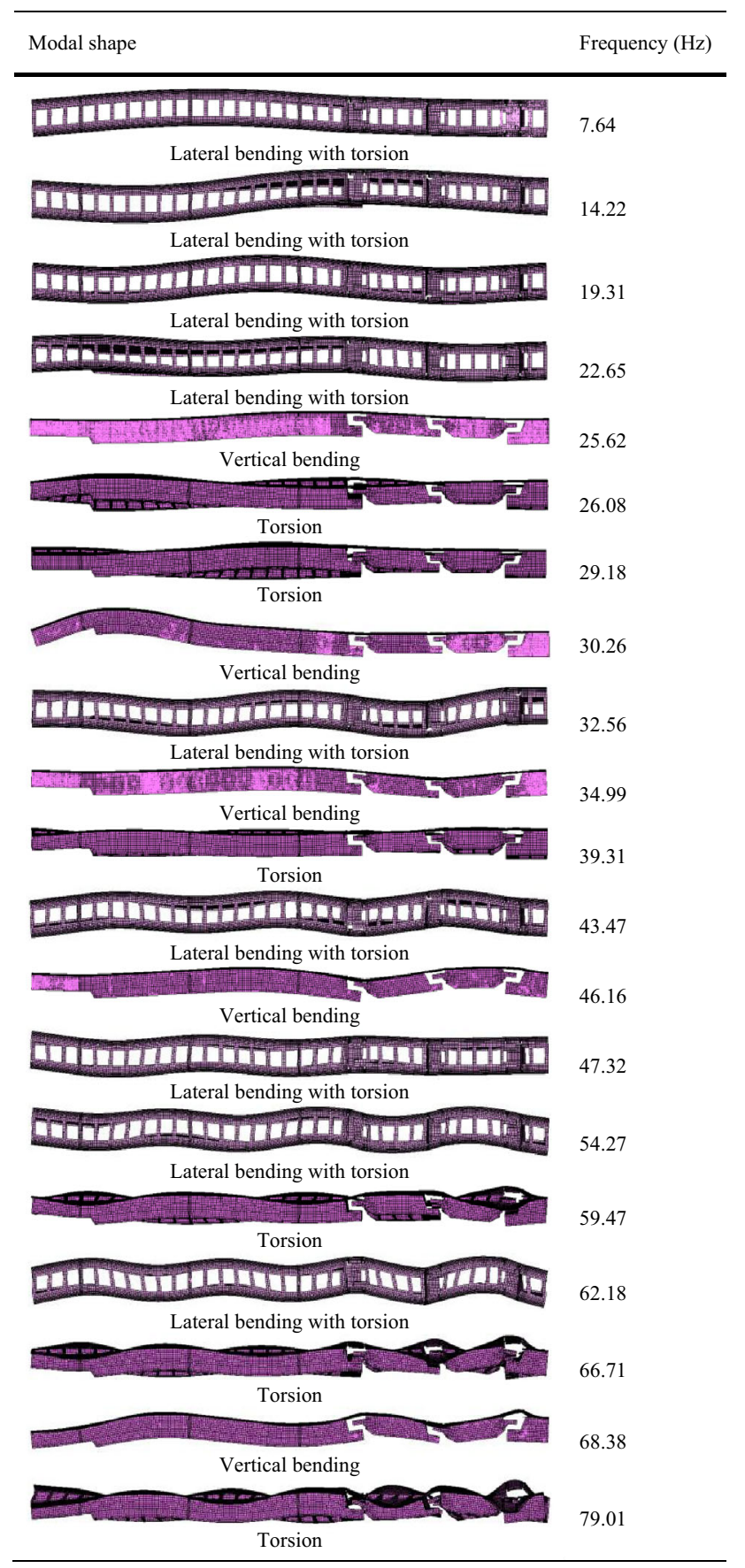

while that in Ref. [40] was $25.03 \mathrm{~Hz}$, both of which are close to the data of this paper. The above results illustrate that although similar turnout structures are used in different test lines and commercially operated lines, there may be large differences in support stiffness of LSG, which in turn gives rise to the fact that the modal results obtained by different researchers vary considerably. 
Table 4 Natural vibration modes of the turnout with 2 trolleys supporting LSG

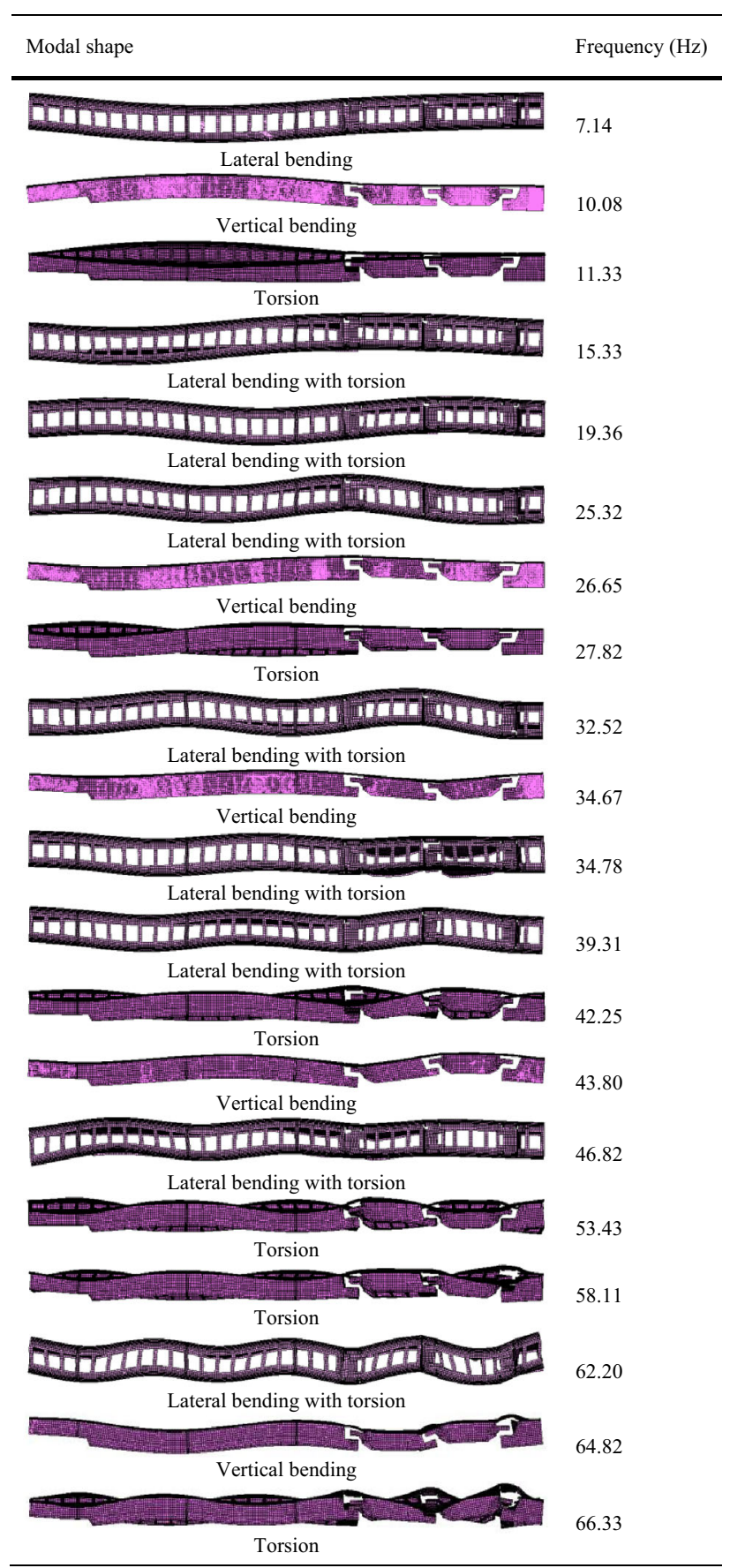

On the whole, the modal simulation results for the turnout are in good agreement with the measured results, so they can be used for subsequent predictive analysis on the dynamic response of the coupled system, including study on severe coupled vibration that occurred before, and also can be used for the assessment of the dynamic stress response of the turnout in service.

\subsection{Dynamic responses of the turnout}

\subsubsection{Dynamic displacement of the LSG}

Figure 9 shows how the maximum vertical dynamic displacement of the 1st and 2nd span of LSG varies with speed when the vehicle with no load runs at different speeds along the main line and auxiliary line, respectively. The suffix of the horizontal coordinates in Fig. 9b and some subsequent figures indicates the test sequence; for example, the ' $10 \_3$ ' means that the test vehicle passed the turnout for the $3 \mathrm{rd}$ time at the speed of $10 \mathrm{~km} / \mathrm{h}$. On the whole, the test results are consistent, indicating the reliability of the data. The maximum value of vertical dynamic displacement of the LSG is not sensitive to changes in vehicle speed. When the vehicle moves straight ahead along the main line, the maximum values of the 1 st and 2 nd span are within the range of $0.440-0.457$ and $0.579-0.597 \mathrm{~mm}$, respectively, while they are within $0.462-0.471$ and $0.531-0.543 \mathrm{~mm}$, respectively, when the vehicle switches to the auxiliary line; the maximum vertical dynamic displacement of the 2nd span is greater than that of the 1st span, which is mainly attributed to the structure of the girder: the support span of the 2nd span (9000 $\mathrm{mm})$ is slightly larger than that of the 1st span $(8819 \mathrm{~mm})$, and the measurement point of vertical dynamic displacement on the 2 nd span is closer to the location where the maximum dynamic displacement occurs. In addition, as CJ/T 412-2012 Technical Specification for Medium and Low Speed Maglev Turnout stipulates that the maximum vertical dynamic displacement of the turnout girder should not be greater than $L / 3800$ ( $L$ is the support span of the turnout girder), the test results show that it meets the requirement of the standard.

\subsubsection{Vibration acceleration of the turnout}

Figures 10 and 11 show the variation of acceleration with speed for each vibration measurement point on the turnout when the vehicle is passing along the main line and auxiliary line, respectively. The vibration acceleration measurement points on the turnout subsystem are handled with band-pass filter having a frequency range of $0.4-150 \mathrm{~Hz}$, based on the analysis of the natural vibration characteristics of the turnout subsystem in Sect. 3.1 and the modal information of LSG and SSG in the literature [27].

According to Fig. 10, the amplitude of the vibration acceleration at each vibration measurement point does not follow an obvious linear pattern as the vehicle speed increases. At all speeds, the vertical acceleration of the 1st span of LSG and SSG \#2 is greater than the corresponding lateral value, while the lateral acceleration of the 2 nd span of LSG and SSG \#1 is almost always higher than the 
(a)

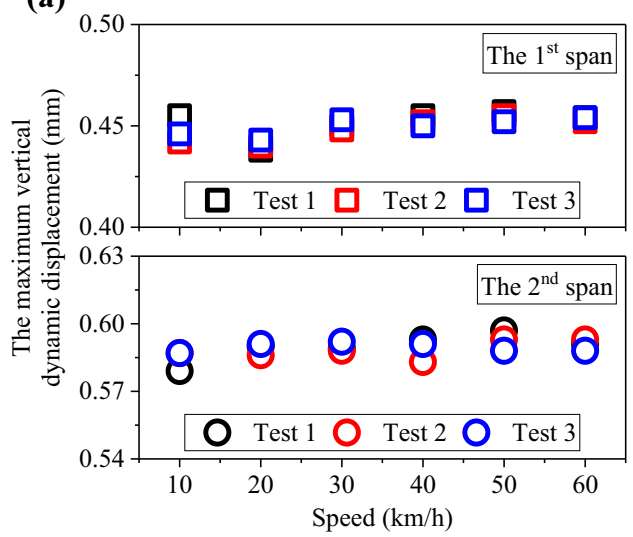

(b)

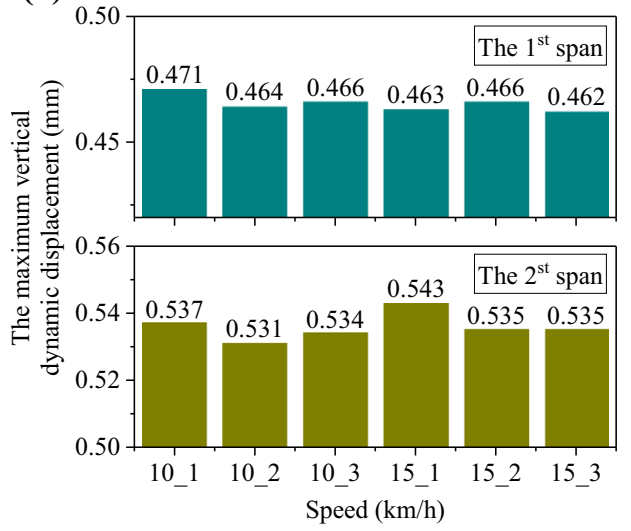

Fig. 9 Variation of the maximum vertical dynamic displacement of LSG with speed: a when the vehicle moves straight ahead along the main line; $\mathbf{b}$ when the vehicle switches to the auxiliary line

(a)

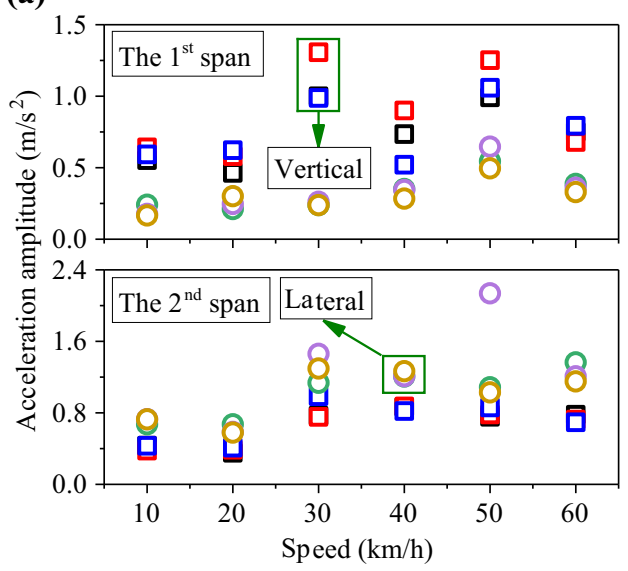

(b)

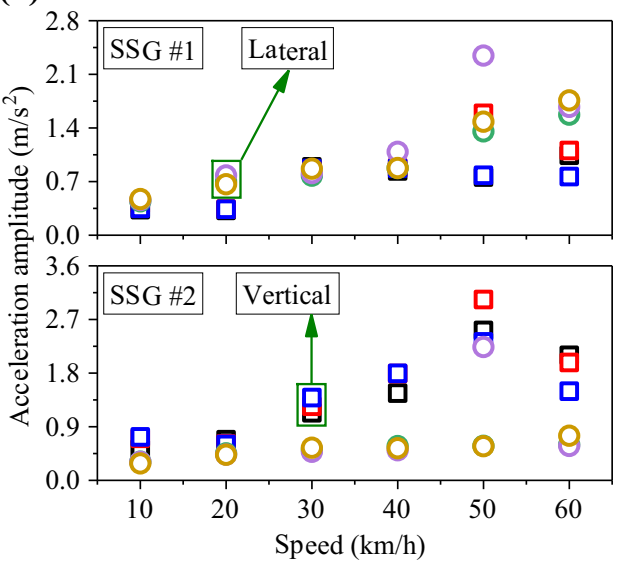

(c)

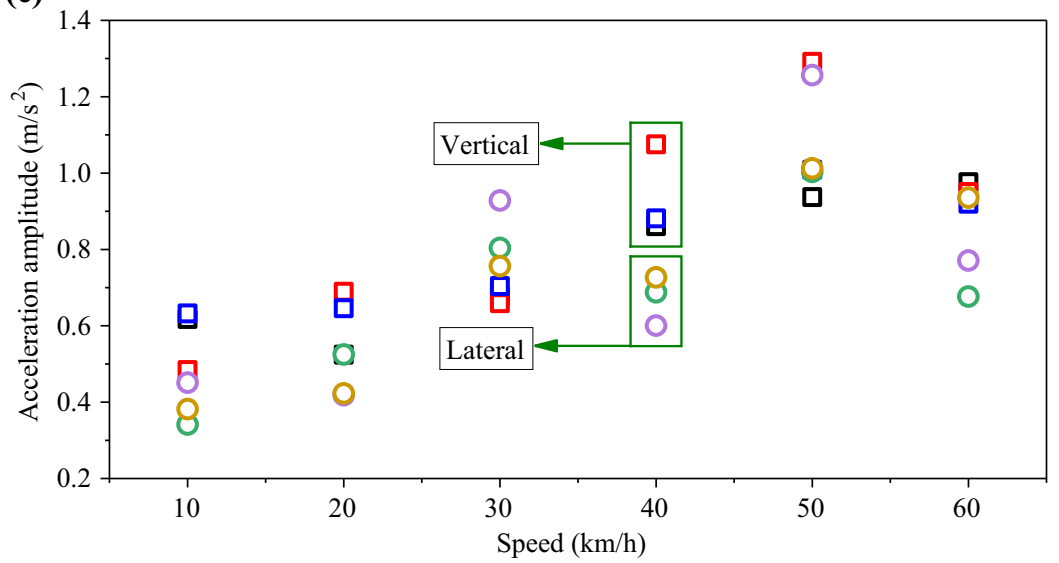

Fig. 10 Variation of acceleration with speed for each vibration measurement point on the turnout subsystem when the vehicle runs along the main line: a LSG; b SSG; $\mathbf{c}$ upper flange

vertical one. At the upper flange, the vertical acceleration is significantly greater, which may be related to the special structure of the turnout subsystem. The vertical accelerations of the 1st span of LSG, the 2nd span of LSG, SSG \#1,
SSG \#2 and upper flange are within the range of $0.463-1.307,0.352-0.985,0.323-1.590,0.528-3.034$ and $0.484-1.291 \mathrm{~m} / \mathrm{s}^{2}$, respectively, while their corresponding lateral acceleration amplitudes are within the range of 
(a)

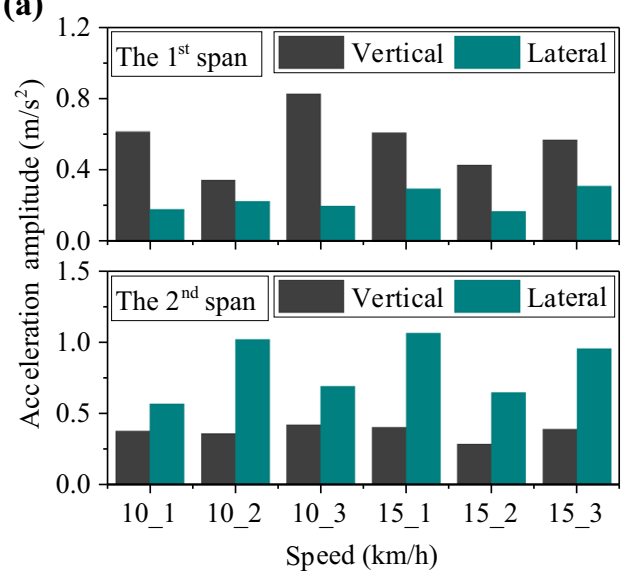

(b)

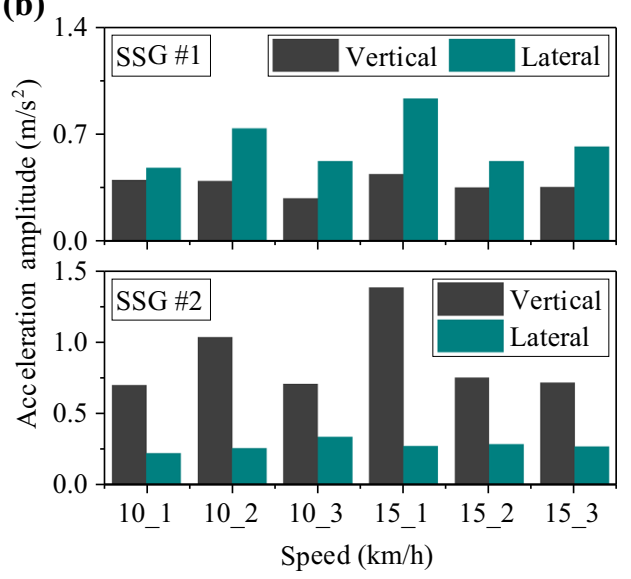

(c)

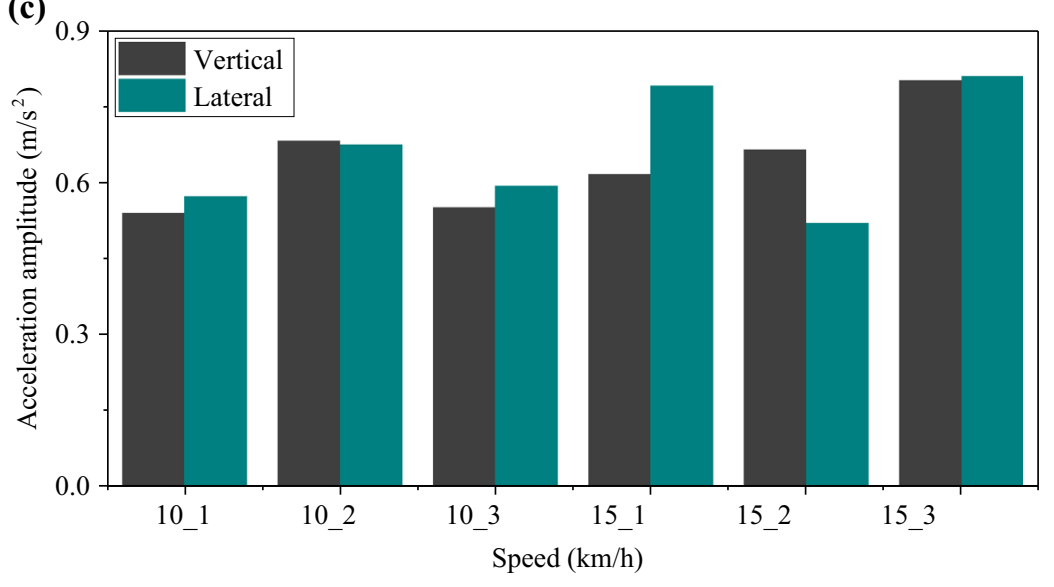

Fig. 11 Variation of acceleration with speed for each vibration measurement point on the turnout subsystem when the vehicle switches to auxiliary line: a LSG; b SSG; $\mathbf{c}$ upper flange

$0.166-0.647,0.578-2.137,0.442-2.344,0.282-0.748$ and $0.341-1.257 \mathrm{~m} / \mathrm{s}^{2}$, respectively.

As shown in Fig. 11, when the vehicle switches to the auxiliary line, the vertical acceleration of the 1st span of LSG and SSG \#2 is greater than the corresponding lateral value, whereas the lateral acceleration of the 2 nd span of LSG and SSG \#1 is higher than the vertical one At the upper flange, the lateral acceleration is significantly greater; the vertical acceleration amplitudes of the 1st span of LSG, the 2nd span of LSG, SSG \#1, SSG \#2 and upper flange are within the range of $0.344-0.828,0.289-0.424$, $0.279-0.438,0.697-1.380$ and $0.540-0.801 \mathrm{~m} / \mathrm{s}^{2}$, respectively, while their corresponding lateral acceleration amplitudes are within the range of $0.170-0.313$, 0.567-1.061, 0.478-0.929, 0.219-0.336 and $0.519-0.810 \mathrm{~m} / \mathrm{s}^{2}$, respectively. As there is a speed limit when the vehicle switches to the auxiliary line, the vibration responses of each measurement point are only tested at speeds of 10 and $15 \mathrm{~km} / \mathrm{h}$. However, it is difficult to maintain the speed exactly at the two values in the field tests, apart from the structural influence of the turnout itself. Therefore, the vibration amplitude of each measurement point shows strong dispersion.

\subsection{Dynamic responses of the vehicle}

\subsubsection{Response of the levitation gap}

To characterize the variation of the levitation gap when the vehicle passes through the turnout (the rated levitation gap is $8.5 \mathrm{~mm}$ ), data at levitation points \#5 and \#7 are obtained via the levitation gap sensor during the test. Figure 12 shows the fluctuations of the levitation gap with speed at levitation points \#5 and \#7 in the vehicle subsystem as the vehicle passes through the turnout along the main line and auxiliary line.

According to Fig. 12, within a certain speed range, the levitation gap fluctuation tends to increase as the vehicle speed increases (when the vehicle passes through the turnout along the main line), and the gap fluctuation of levitation point \#7 is larger than that of \#5, indicating that the levitation controller corresponding to $\# 5$ has better 
(a)

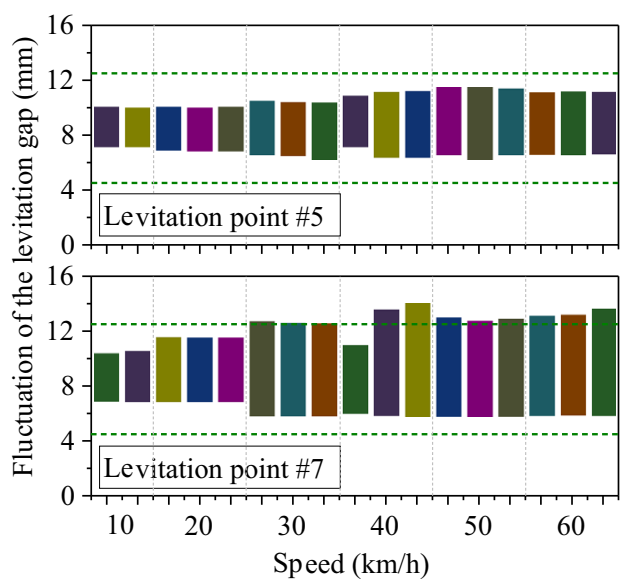

(b)

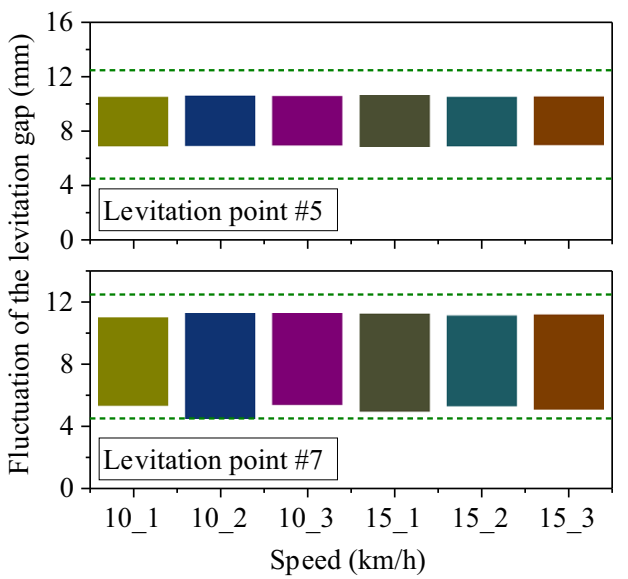

Fig. 12 Fluctuation of the levitation gap: a when the vehicle moves straight ahead along the main line; $\mathbf{b}$ when the vehicle switches to the auxiliary line

performance. When the vehicle moves straight ahead, the levitation gap at points \#5 and \#7 is within 6.22-11.46 and $5.76-13.98 \mathrm{~mm}$, respectively, whereas it is within 6.87-10.64 and 4.49-11.25 $\mathrm{mm}$ when the vehicle switches to the auxiliary line. Although levitation point \#7 does not lose levitation stability or contact the track at various speeds, it fails to meet this requirement of $C J / T$ 458-2014 Technical Specification of System for the Levitation Control of Medium-low Speed Maglev Transportation Vehicle, which stipulates that the deviation of the levitation gap during dynamic operation of the train should be within $\pm 4 \mathrm{~mm}$ (especially when the vehicle passes the turnout along the main line at $30-60 \mathrm{~km} / \mathrm{h}$ ). Therefore, to have a stable levitation gap fluctuation range when passing the turnout, it is recommended to check the installation accuracy of the levitation gap sensor at point \#7.

\subsubsection{Vibration acceleration of the vehicle}

To investigate the vibration characteristics of the levitation frame when the vehicle passes through the turnout, vertical and lateral acceleration sensors are arranged on the corresponding brackets of levitation points \#5 and \#7, respectively, and the obtained dynamic response signals are handled with band-pass filter having a frequency range of $0.4-100 \mathrm{~Hz}$. Figure 13 shows how the vibration acceleration of the brackets varies with speed when the vehicle passes through the turnout along the main line and auxiliary line. As shown in Fig. 13a, the consistency of the lateral acceleration of the front-end and rear-end brackets in the three tests is better than that in the vertical direction at all speeds, mainly because it is difficult to keep the vehicle running at strictly the same speed during the test and because the effect on the dynamic response of the vertical direction is relatively greater when the vehicle passes through the turnout in the straight direction; on the whole, the vibration acceleration of the brackets increases with speed and is higher at the rear end than at the front end due to the greater levitation gap fluctuation at the point \#7. The vertical acceleration of the front- and rear-end brackets is within the range of $2.893-7.761$ and $10.105-27.287 \mathrm{~m} / \mathrm{s}^{2}$, respectively, and their corresponding lateral acceleration is within $1.786-3.045$ and $2.773-6.342 \mathrm{~m} / \mathrm{s}^{2}$, respectively.

According to Fig. 13b, when the vehicle switches to the auxiliary line, the lateral acceleration of both the front- and rear-end brackets is lower than that in the vertical direction, mainly due to the low running speed (no more than $15 \mathrm{~km} /$ h), which makes the passive lateral impact not obvious; besides, the performance of controller at point \#7 is worse than that at point \#5, with greater differences in lateral and vertical accelerations; the vertical acceleration of the frontand rear-end brackets is within the range of 3.706-6.331 and $12.230-21.978 \mathrm{~m} / \mathrm{s}^{2}$, respectively, and the corresponding lateral acceleration is within 1.918-3.923 and $2.955-5.103 \mathrm{~m} / \mathrm{s}^{2}$, respectively.

The dynamic response characteristics of the car body are closely related to the passenger's riding comfort. With reference to GB/T 5599-2019 Specification for Dynamic Performance Assessment and Testing Verification of Rolling Stock for lateral and vertical ride quality, the vibration test results of the car body are handled with band-pass filter having frequency range of $0.4-40 \mathrm{~Hz}$. Figure 14 presents the variation of the vibration acceleration of the car body with speed when the vehicle passes the turnout along the main and auxiliary lines. Overall, the vibration acceleration in the middle and at the rear end of the car body does not exceed $2.5 \mathrm{~m} / \mathrm{s}^{2}$ regardless of the running direction, which meets the requirement in GB/T 5599-2019, indicating that 
(a)

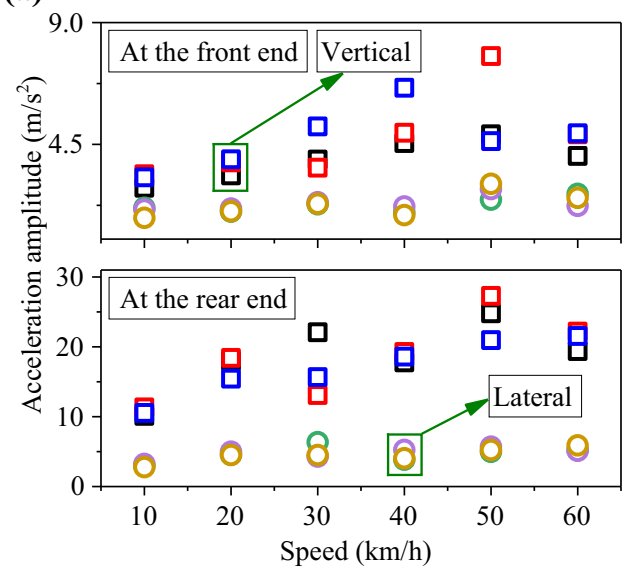

(b)

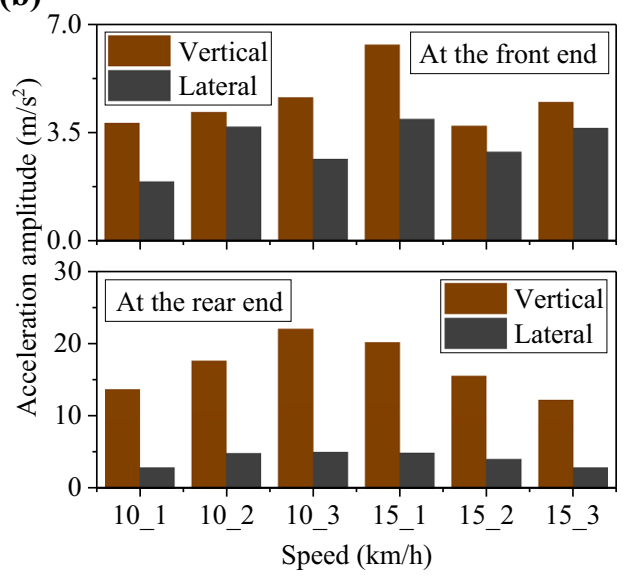

Fig. 13 Variation of the vibration acceleration of the brackets with speed: a when the vehicle moves straight ahead along the main line; $\mathbf{b}$ when the vehicle switches to the auxiliary line

(a)

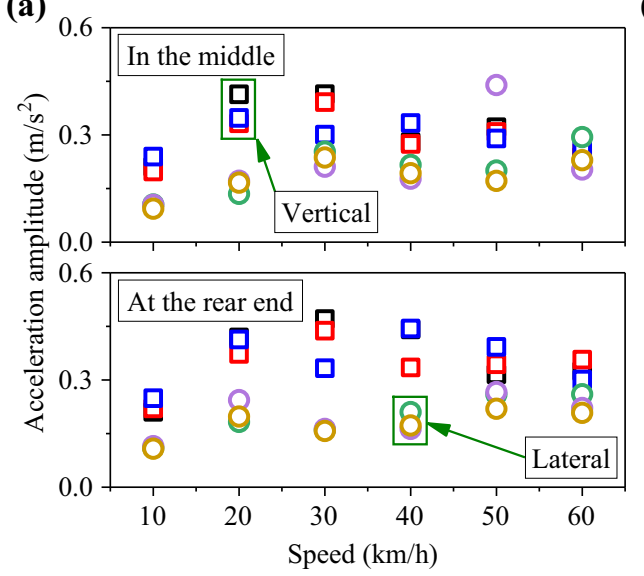

(b)

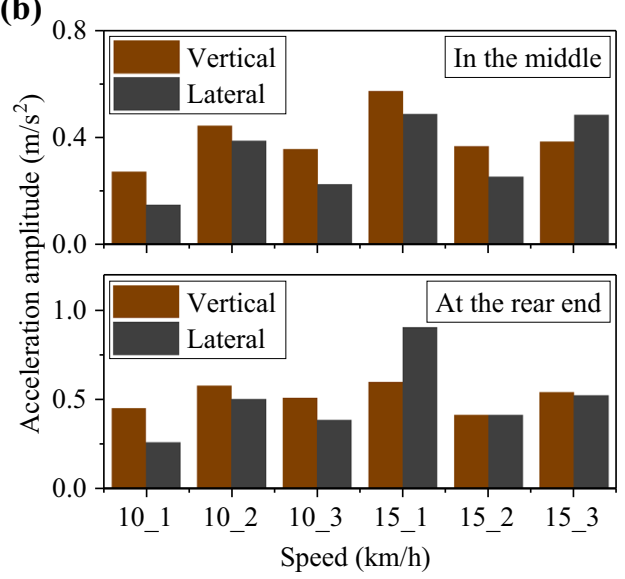

Fig. 14 Variation of the vibration acceleration of the car body with speed: $\mathbf{a}$ when the vehicle moves straight ahead along the main line; $\mathbf{b}$ when the vehicle switches to the auxiliary line

the vehicle has good ride quality when passing through the turnout, despite the poor control performance of the levitation point \#7.

As shown in Fig. 14a, the vertical acceleration both in the middle and at the rear-end measurement points is greater than the respective lateral acceleration (except for the results of the $2 \mathrm{nd}$ test at the speed of $50 \mathrm{~km} / \mathrm{h}$ ), mainly because the vertical direction plays a dominant role and the lateral disturbance is relatively small when running in the straight direction; besides, the vertical acceleration at the rear end of the car body is almost greater than that in the middle, which may be caused by the pitching motion of the vehicle. Specifically, the vertical and lateral accelerations in the middle of the car body are within the range of $0.197-0.414$ and $0.094-0.440 \mathrm{~m} / \mathrm{s}^{2}$, respectively, and those in the rear end are within $0.211-0.470$ and $0.107-0.266 \mathrm{~m} /$ $\mathrm{s}^{2}$, respectively. Figure $14 \mathrm{~b}$ shows that the vertical and lateral accelerations in the middle of the car body are within the range of $0.273-0.574$ and $0.150-0.488 \mathrm{~m} / \mathrm{s}^{2}$, respectively, and those in the rear end is within $0.414-0.598$ and $0.261-0.904 \mathrm{~m} / \mathrm{s}^{2}$, respectively, indicating that both vertical and lateral vibrations are more affected than when passing the turnout in a straight direction. Therefore, it is recommended to choose a reasonable running speed when the vehicle switches to the auxiliary line. Furthermore, Fig. 14a shows that the vertical acceleration of the vehicle peaks at $30 \mathrm{~km} / \mathrm{h}$; it increases with increasing speed within $10-30 \mathrm{~km} / \mathrm{h}$, and the increase is even greater from 10 to $20 \mathrm{~km} / \mathrm{h}$. However, in the speed range of $30-60 \mathrm{~km} / \mathrm{h}$, there is an opposite trend, yet the change is relatively slight, which may be related to factors such as the levitation control system and the vibration isolation capacity of the air springs. 
(a)

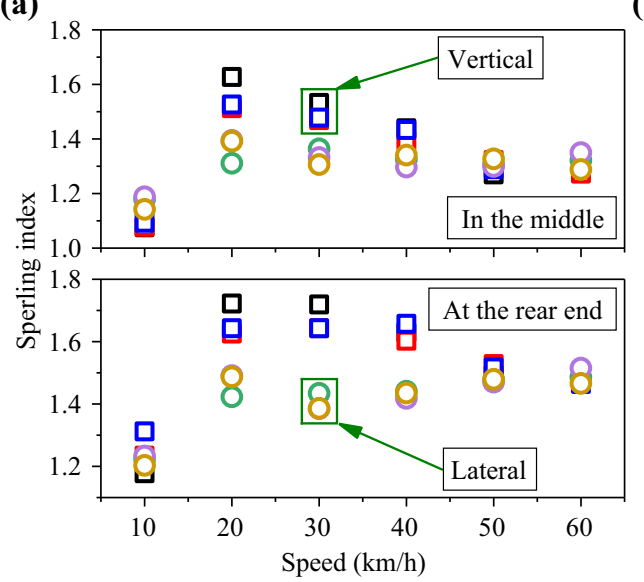

(b)

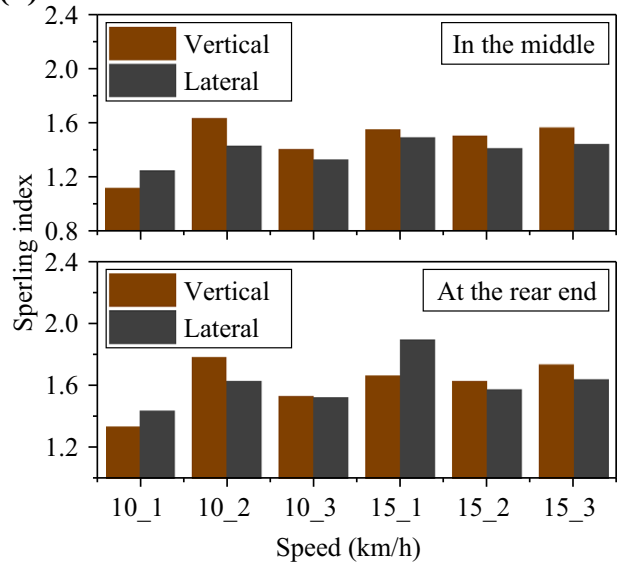

Fig. 15 Variation of the Sperling index with speed: a when the vehicle moves straight ahead along the main line; $\mathbf{b}$ when the vehicle switches to the auxiliary line

\subsubsection{Ride quality of the vehicle}

To further evaluate the ride quality of the vehicle when passing the turnout, Sperling indexes of the two measurement points in the middle and at the rear end of the car body are calculated under each running condition according to the method in Ref. [41] (see Eq. (1)), using the time domain data of the vibration response obtained from the actual measurements. The variation of the Sperling index with speed when the vehicle passes through the turnout is shown in Fig. 15. It can be seen that when the vehicle passes the turnout along the main line, the maximum values of the vertical and lateral Sperling index in the middle of the car body are 1.627 and 1.395 , respectively, with corresponding values of 1.722 and 1.515 at the rear end. When the vehicle switches to the auxiliary line, the corresponding index is 1.638 and 1.497 in the middle of the car body and 1.782 and 1.894 at the rear end. This reflects that the vehicle has relatively poor vertical and lateral ride quality when switching to the auxiliary line; however, the vertical and lateral Sperling index in the middle and rear end of the car body does not exceed 2.5 regardless of the running direction, indicating the excellent ride quality of the vehicle.

$W_{i}=7.08 \times \sqrt[10]{A_{i}^{3} F\left(f_{i}\right) / f_{i}}$,

where $W_{i}$ is the component of the Sperling index at the frequency $f_{i}(i=1,2, \ldots, n) ; A_{i}$ is vibration acceleration of car body $(\mathrm{g}) ; f_{i}$ is the vibration frequency $(\mathrm{Hz})$, $0.5 \mathrm{~Hz} \leq f_{i} \leq 40 \mathrm{~Hz} ;$ and $F\left(f_{i}\right)$ is the frequency
Table 5 Frequency correction coefficient (GB/T 5599-2019)

\begin{tabular}{lllll}
\hline Vertical vibration & & & \multicolumn{2}{c}{ Lateral vibration } \\
\cline { 1 - 1 }$f_{i}(\mathrm{~Hz})$ & $F\left(f_{i}\right)$ & & $f_{i}(\mathrm{~Hz})$ & $F\left(f_{i}\right)$ \\
\hline $0.5-5.9$ & $0.325 f_{i}^{2}$ & & $0.5-5.4$ & $0.8 f_{i}^{2}$ \\
$5.9-20$ & $400 / f_{i}^{2}$ & & $5.4-26$ & $650 / f_{i}^{2}$ \\
$\geq 20$ & 1 & $\geq 26$ & 1 \\
\hline
\end{tabular}

correction coefficient, as shown in Table 5. Finally, the total Sperling index $W$ is given by

$W=\sqrt[10]{\sum_{i=1}^{n} W_{i}^{10}}$

\subsection{Analysis on the spectrum of the vehicle-turnout coupled system}

To further investigate the vibration characteristics of the vehicle-turnout coupled system, fast Fourier transform is performed to convert the time history of vibration acceleration response. The acceleration frequency domains for the turnout subsystem and the vehicle subsystem in the vertical and lateral directions are presented in Figs. 16 to 19 , respectively.

Figures 16 and 17 show that the peak frequencies of the LSG and SSGs in both the vertical and lateral directions are above $20 \mathrm{~Hz}$ when the vehicle passes through the turnout at different speeds, and multiple dominant bands exist for 
both; the speed of the vehicle and the direction have no significant effect on the peak vibration frequency range of the turnout subsystem; and the dominant frequency band of vertical vibration of LSG is mainly concentrated within $20-40 \mathrm{~Hz}$. Combined with the analysis on the natural vibration characteristics of the turnout subsystem in Sect. 3.1, it can be further deduced that the dominant natural frequency for the vertical bending vibration of LSG is in the range of $27-30 \mathrm{~Hz}$; the 1st span of LSG and SSG \#2 have higher vertical acceleration than lateral acceleration in the dominant frequency band, while the 2nd span of LSG and SSG \#1 have almost higher lateral acceleration, indicating that under the same external excitation, the 1st span of LSG and SSG \#2 have similar vibration characteristics, so do the 2nd span of LSG and SSG \#1. As a result, the vertical acceleration of the 1st span of LSG and SSG \#2 is greater than the corresponding lateral acceleration in all operating conditions, while the lateral vibration of the 2nd span of LSG and SSG \#1 is higher (see Sect. 3.2.2).

(a)

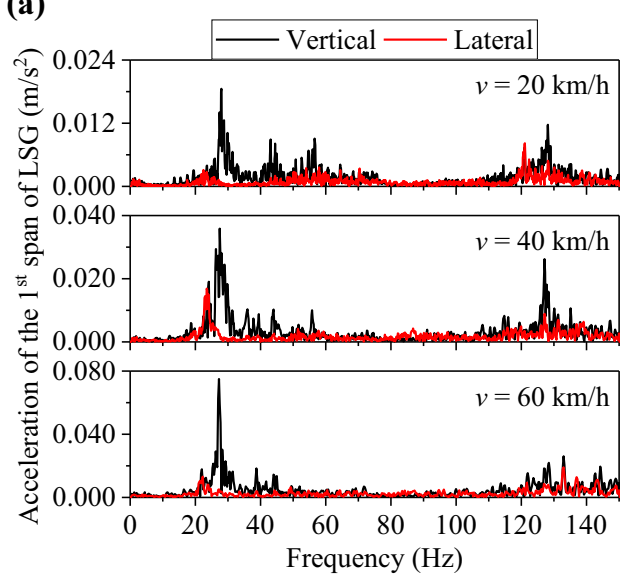

(c)

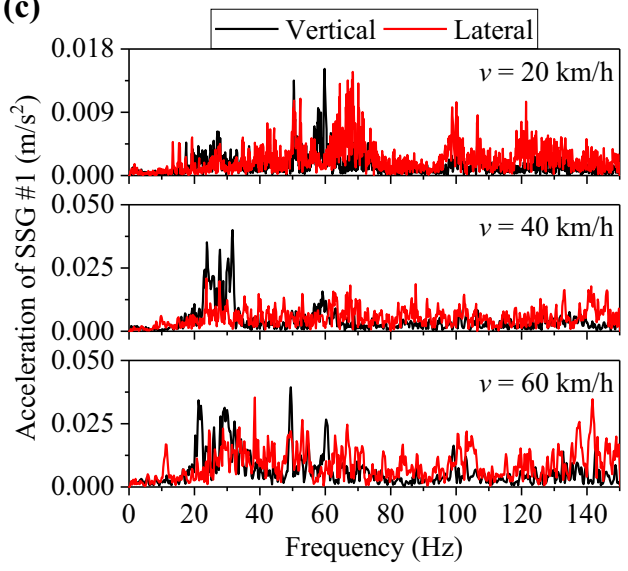

Furthermore, as shown in Fig. 16c, d and Fig. 17c, d, the dominant frequency bands for the vertical acceleration of SSG \#1 are $20-35$ and $50-60 \mathrm{~Hz}$, while the dominant frequency distribution for its lateral acceleration is broader, with multiple peak frequencies in the range of $20-150 \mathrm{~Hz}$. The peak vertical acceleration frequency of SSG \#2 is mainly concentrated around $34 \mathrm{~Hz}$, which is generally consistent with what is shown in Fig. 7b; that is, SSG \#2 is mainly controlled by the frequency of $34 \mathrm{~Hz}$ within $80 \mathrm{~Hz}$, reflecting its natural vibration characteristics. In addition, the lateral acceleration of SSG \#2 also has a wide range of dominant frequencies, and it is mainly controlled by the frequency band of $45-75 \mathrm{~Hz}$.

As shown in Fig. 18a and Fig. 19a, when the vehicle is running at a low speed (no more than $20 \mathrm{~km} / \mathrm{h}$ ), the vertical and lateral accelerations of the front-end bracket is small; when the speed is increased, its vibration acceleration is mainly controlled by a low-frequency band within $10 \mathrm{~Hz}$. Figures 18a, b and 19a, b show that under the effect of the dominant frequency band, the vibration acceleration of the

(b)

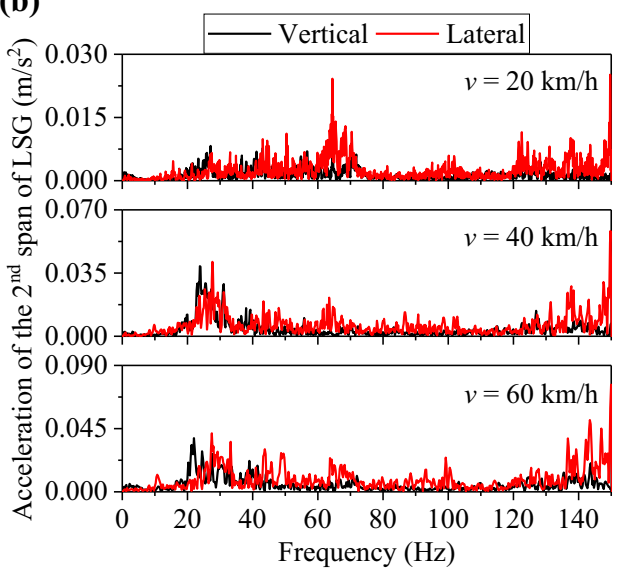

(d)

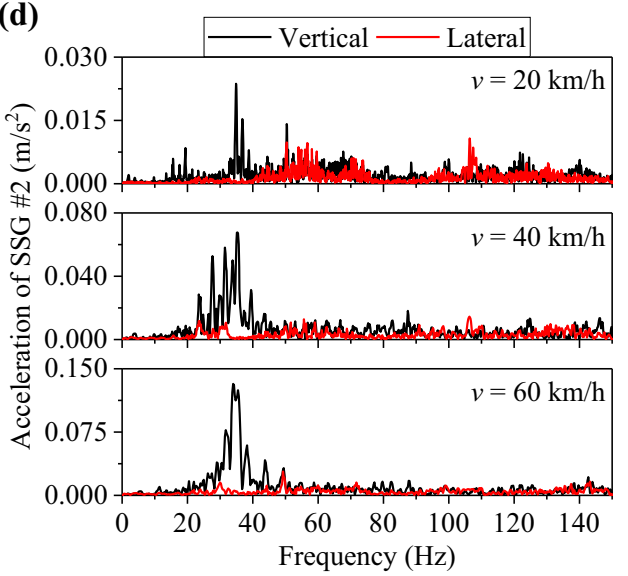

Fig. 16 Vibration acceleration spectra of turnout subsystem when the vehicle moves straight ahead along the main line: a the 1st span of LSG; b the 2nd span of LSG; c SSG \#1; d SSG \#2 
(a)

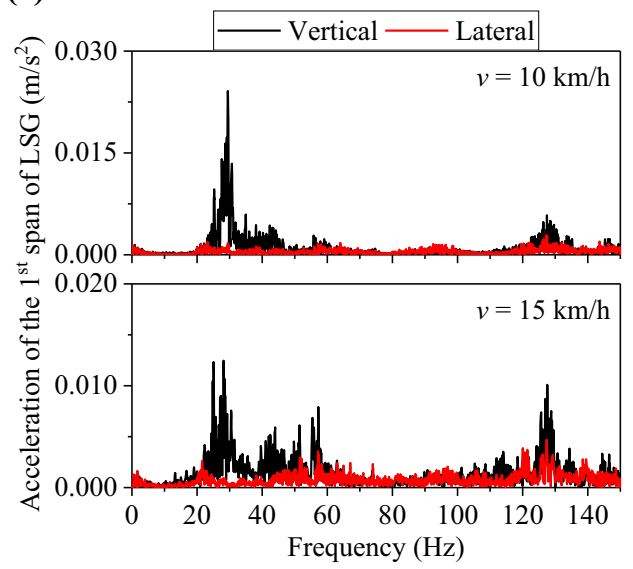

(c)

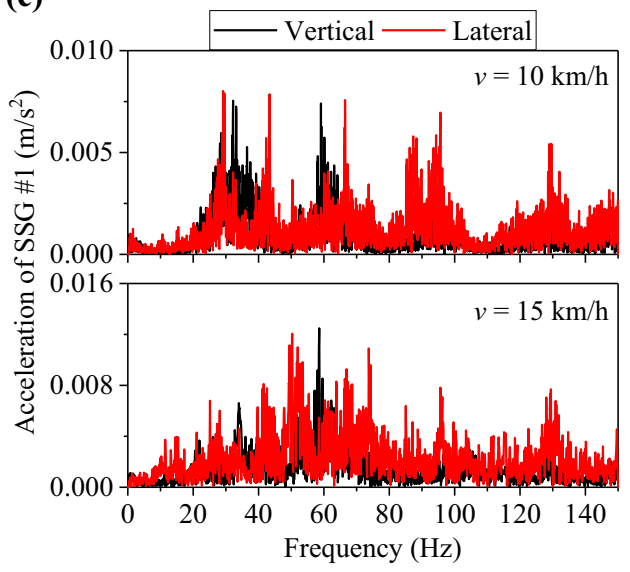

(b)

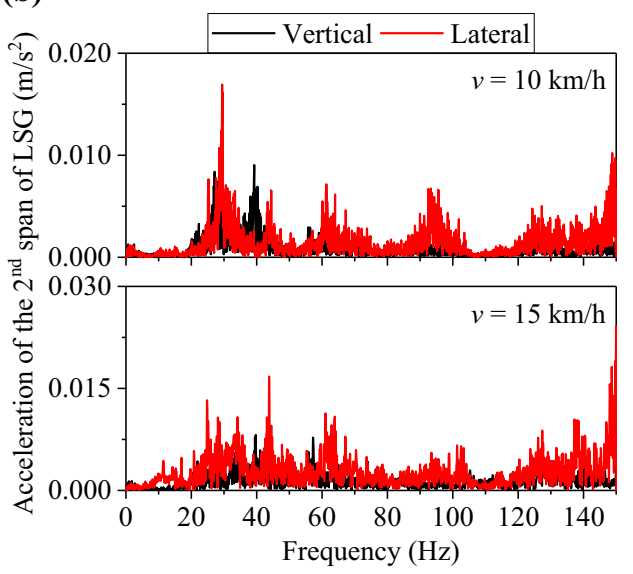

(d)

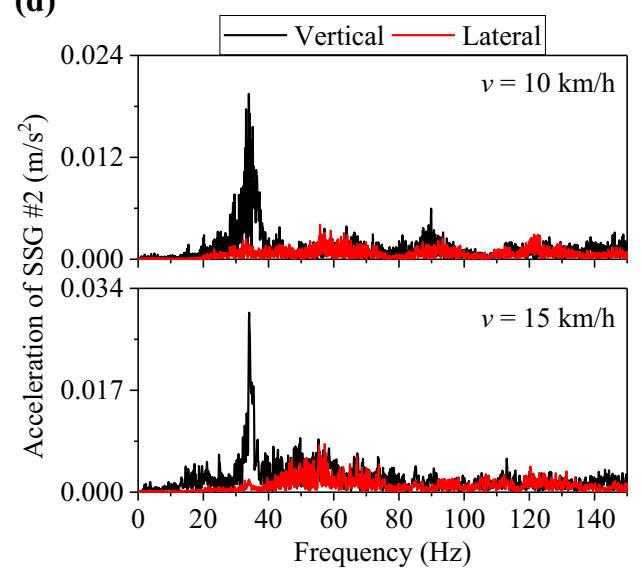

Fig. 17 Vibration acceleration spectra of turnout subsystem when the vehicle switches to the auxiliary line: a the 1st span of LSG; b the 2nd span of LSG; c SSG \#1; d SSG \#2

rear-end bracket of the tested levitation module is greater than that of the front end, as the position of the rear-end bracket corresponds to levitation point \#7, where the levitation gap fluctuation is greater than at point \#5 (see Fig. 12); besides, the rear-end bracket has a lower-frequency vibration (below $1 \mathrm{~Hz}$ ) compared to the front-end bracket, while having a much larger vibration compared to point \#5, which may be related to the integral initial drift within the levitation controller corresponding to this measurement point.

According to Figs. 18c, d and $19 \mathrm{c}$, d, due to the vibration isolation of the air springs, some of the frequency components from the levitation frame are effectively separated, resulting in the relatively smaller vibration acceleration in the car body. When the vehicle passes straight along the main line, the vertical acceleration is greater than the lateral acceleration under the effect of dominant frequencies below $5 \mathrm{~Hz}$. When the vehicle switches to auxiliary line at 10 and $15 \mathrm{~km} / \mathrm{h}$, the lateral acceleration is higher under the effect of dominant frequencies below $5 \mathrm{~Hz}$ in almost all conditions, because the car body is subjected to a certain degree of lateral disturbance at this moment. Besides, the dominant vibration frequency component in the vertical direction is present around $38.5 \mathrm{~Hz}$ both in the middle and at the rear end of the car body and it is not subjected to the influence of vehicle speed or running direction, reflecting the natural vibration characteristics of the car body. The frequency of $38.5 \mathrm{~Hz}$ may be related to the electrical equipment hanging under the vehicle. Overall, the bracket and car body are mainly controlled by the frequency band below $10 \mathrm{~Hz}$.

\subsection{Vehicle standing still above the LSG}

To clarify the dynamic response characteristics of the coupled system when the vehicle is standing still above the LSG, Figs. 20, 21 and 22 show the levitation gap and the dynamic response of the vertical acceleration of each 

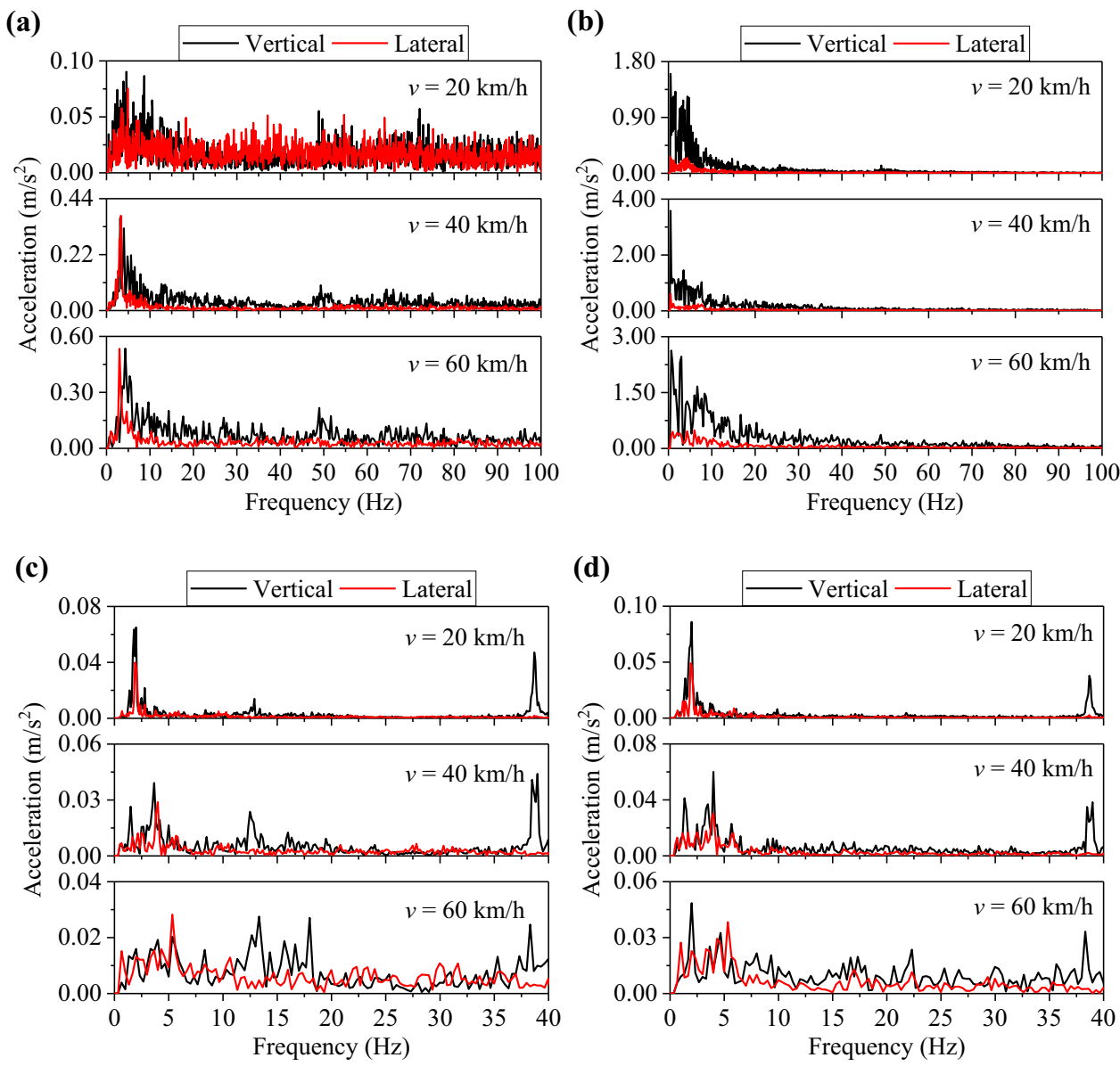

Fig. 18 Vibration acceleration spectra of vehicle subsystem when the vehicle moves straight ahead along the main line: a front-end bracket; b rear-end bracket; $\mathbf{c}$ middle of the car body; $\mathbf{d}$ rear end of the car body

subsystem, respectively. According to Fig. 20, the levitation gap at point \#5 is smaller than that at point \#7, due to factors such as the structural accuracy of the levitation gap sensors and static track irregularity of the turnout subsystem; gap fluctuations at both points are small and basically do not exceed $\pm 0.05 \mathrm{~mm}$, indicating that the vehicle has excellent levitation stability when standing still above the LSG. Thanks to the small gap fluctuation, the fluctuation of the vertical acceleration for the 1st and 2nd spans is also small, not exceeding $\pm 0.03 \mathrm{~m} / \mathrm{s}^{2}$, as shown in Fig. $21 \mathrm{a}$, b. Figure $21 \mathrm{c}, \mathrm{d}$ shows that the vertical acceleration frequency distribution of LSG in the case of standing still is not as characteristic as when the vehicle is passing through. There are local dominant vibration frequencies in the range of 26.7 to $30 \mathrm{~Hz}$ for the 1st span and 2nd span of LSG, but the dominant and local dominant vibration frequencies contribute little to the vibration acceleration of LSG.
Furthermore, Fig. 22a, c shows that the fluctuation of the vertical acceleration of the bracket is about $\pm 1.5 \mathrm{~m} / \mathrm{s}^{2}$; the vertical acceleration of the front-end bracket is dominated by the frequency of $87.3 \mathrm{~Hz}$. The rear-end bracket, however, in addition to having similar high-frequency characteristics to the front-end bracket, also exhibits significant ultra-low-frequency vibration characteristics $(0.4 \mathrm{~Hz})$, which, as described in Sect. 3.4, may be related to the integral initial drift within the levitation controller corresponding to this measurement point. Besides, the high-frequency component of the vertical vibration of the brackets may be related to the brake clamp on the pole plate. Figure $22 \mathrm{~b}, \mathrm{~d}$ shows that the fluctuation of the vertical acceleration of the car body is about $\pm 0.15 \mathrm{~m} / \mathrm{s}^{2}$, and the middle and end of the car body both exhibit similar vibration waveforms; the dominant frequency of vertical vibration of the car body when the vehicle is standing still 
(a)

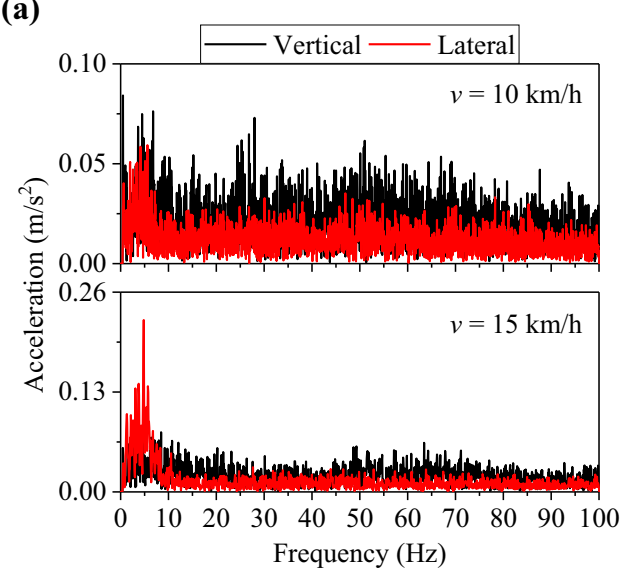

(c)

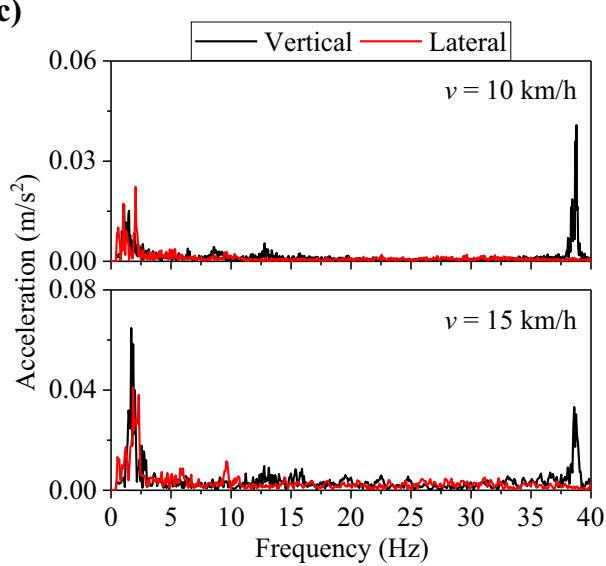

(b)

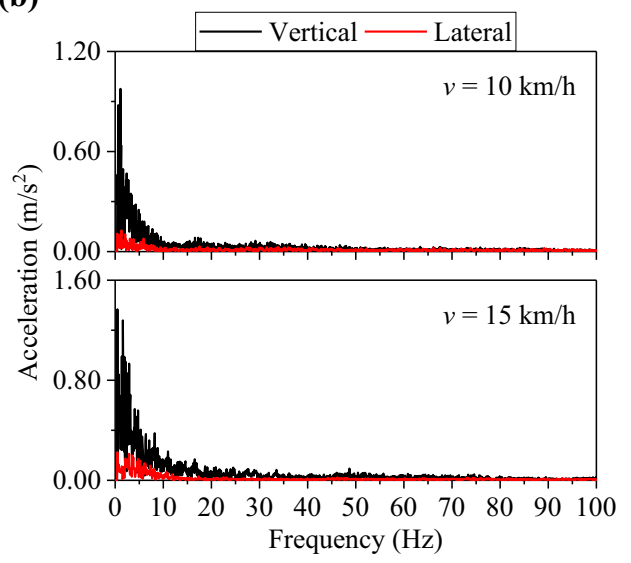

(d)

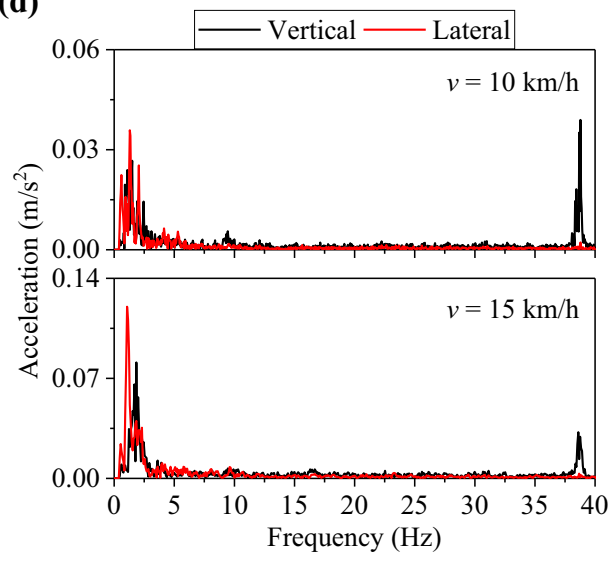

Fig. 19 Vibration acceleration spectra of vehicle subsystem when the vehicle switches to the auxiliary line: a front-end bracket; $\mathbf{b}$ rear-end bracket; $\mathbf{c}$ middle of the car body; $\mathbf{d}$ rear end of the car body

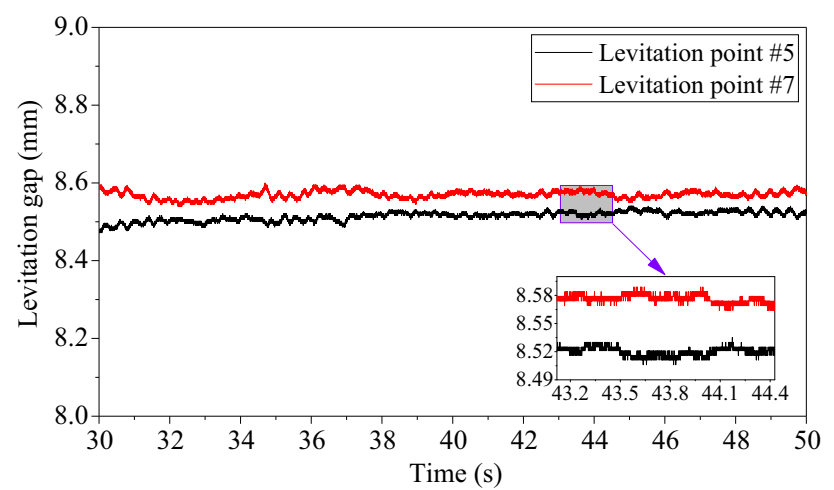

Fig. 20 Time history of levitation gap when the vehicle is standing still above the LSG

above the LSG is around $38.5 \mathrm{~Hz}$, and meanwhile, the car body is also controlled by a locally dominant frequency band below $3 \mathrm{~Hz}$, which contributes relatively less to the amplitude. Combining Figs. 18 and 19, we can see that there is a dominant vibration frequency component around $38.5 \mathrm{~Hz}$ when the vehicle is standing still and running, which is consistent with the results of the correlated analysis in Sect. 3.4.

\section{Conclusions}

With a medium-low-speed maglev test line selected, dynamic response characteristics of the empty vehicleturnout coupled system are analysed in detail in both the time and frequency domains according to the measured data. The main conclusions are listed below.

(1) The turnout subsystem is rich in vibration modes, with the vertical bending natural frequency of LSG in the range of about $27-30 \mathrm{~Hz}$ and the lateral bending vibration of LSG at $14.2 \mathrm{~Hz}$, accompanied by torsional vibration; the SSG, on the other hand, due to its shorter support span, has more dominant frequency components above $40 \mathrm{~Hz}$;

(2) Neither the vertical nor the lateral Sperling index of the vehicle exceeds 2.5 , indicating excellent ride quality under all tested conditions. 
(a)

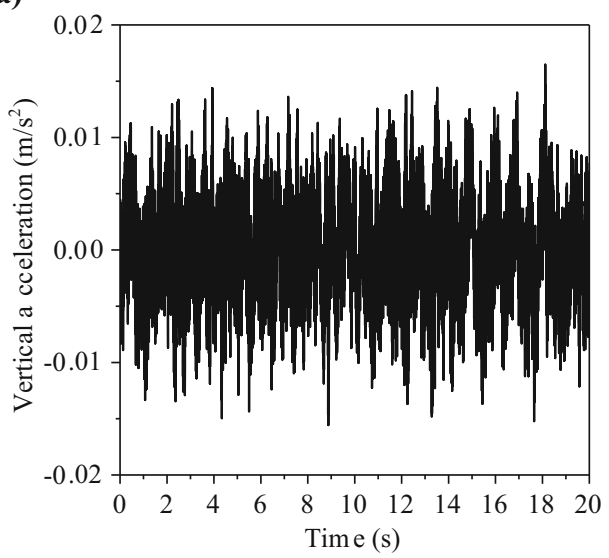

(c)

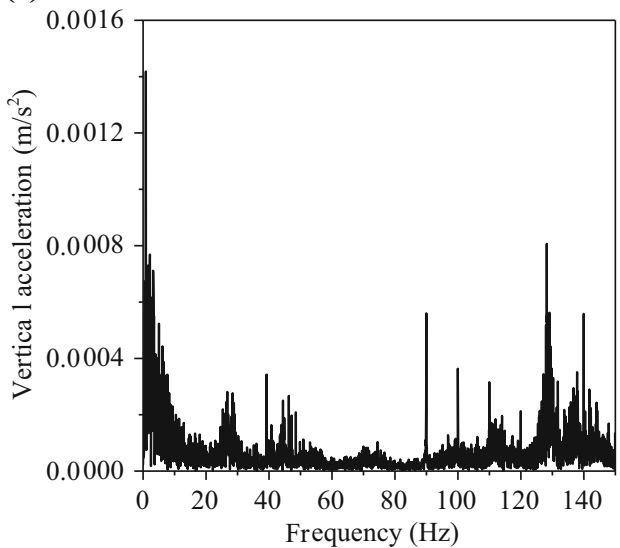

(b)

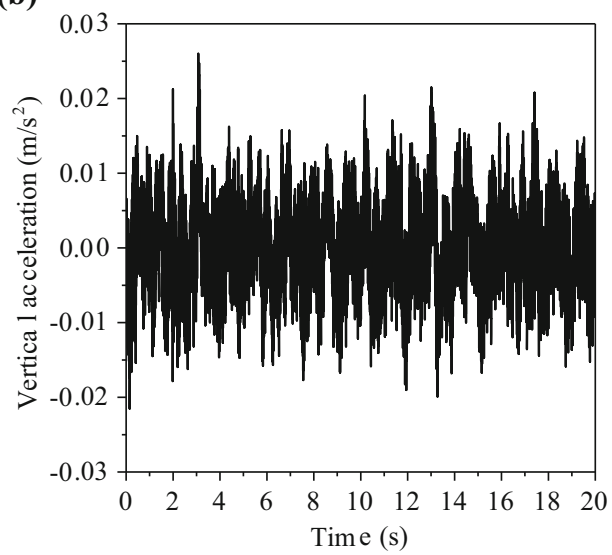

(d)

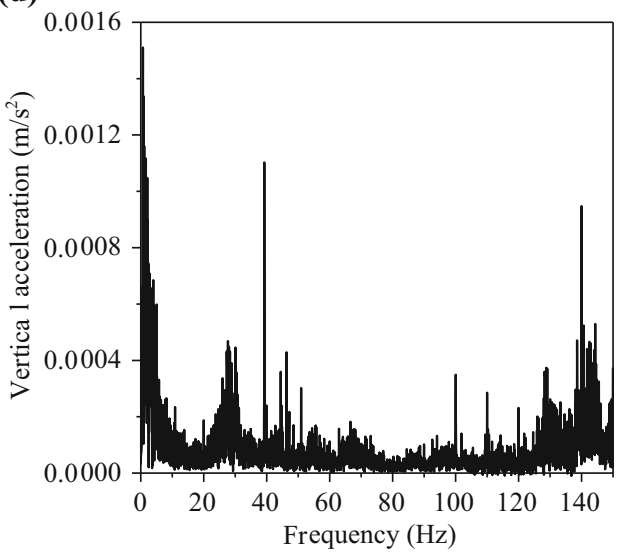

Fig. 21 Time and frequency domains of the vertical acceleration of LSG: a time domain of the 1st span; b time domain of the 2nd span; c frequency domain of the 1st span; $\mathbf{d}$ frequency domain of the 2 nd span

(3) The maximum vertical dynamic displacement of the LSG does not exceed $0.6 \mathrm{~mm}$ and it is not sensitive to the vehicle speed; when the vehicle is in the curved position, the load distribution of the whole vehicle is affected, with a slight increase in the vertical dynamic displacement of the 1st span of LSG and a slight decrease in the 2nd span compared with when the vehicle passes the turnout along the main line.

(4) The vertical dynamic responses of the 1st span of LSG and SSG \#2 are more dramatic than the lateral responses, while the opposite is true for the 2 nd span of LSG and SSG \#1; the lower speed when the vehicle is switching to the auxiliary line results in a smaller maximum vibration response at each measurement point compared with when the vehicle passes the turnout along the main line.

(5) The dominant frequency bands of vertical and lateral acceleration of the car body and bracket are mainly concentrated below $10 \mathrm{~Hz}$ and are characterized by low-frequency vibrations; besides, there is still a local vibration frequency around $38.5 \mathrm{~Hz}$ in the vertical direction, and the existence of other vibration frequencies should be considered in the future optimization of the vehicle design; the peak frequencies of vertical and lateral acceleration vibrations of the turnout subsystem are concentrated above $20 \mathrm{~Hz}$ and 
(a)

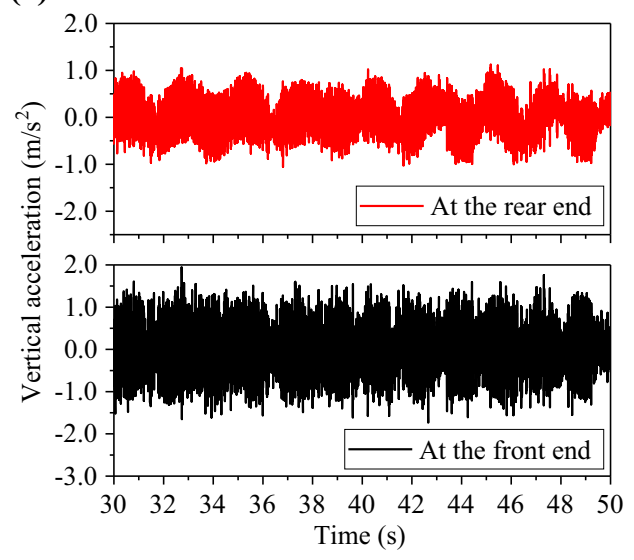

(c)

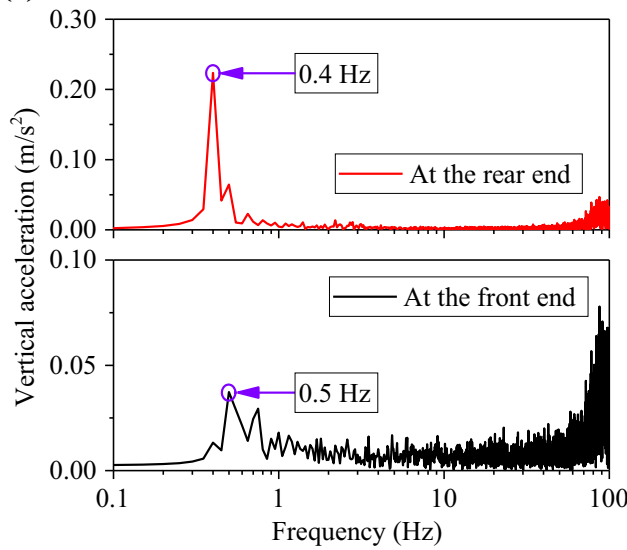

(b)

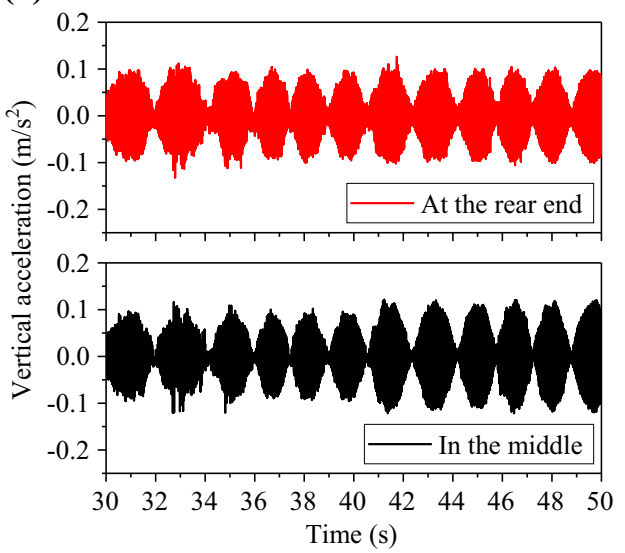

(d)

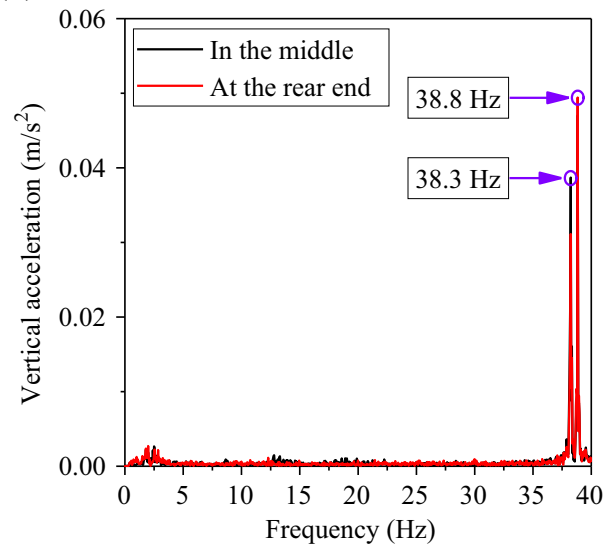

Fig. 22 Time and frequency domains of the vertical acceleration of the vehicle subsystem: a time domain of the bracket; $\mathbf{b}$ time domain of the car body; $\mathbf{c}$ frequency domain of the bracket; $\mathbf{d}$ frequency domain of the car body

are characterized by medium- to high-frequency vibrations.

Acknowledgements This work was supported by the National Natural Science Foundation of China (Grant No. 51875483) and the Independently Funded Research Project of State Key Laboratory of Traction Power (Grant Nos. 2020TPL-T01 and 2020TPL-T04).

Open Access This article is licensed under a Creative Commons Attribution 4.0 International License, which permits use, sharing, adaptation, distribution and reproduction in any medium or format, as long as you give appropriate credit to the original author(s) and the source, provide a link to the Creative Commons licence, and indicate if changes were made. The images or other third party material in this article are included in the article's Creative Commons licence, unless indicated otherwise in a credit line to the material. If material is not included in the article's Creative Commons licence and your intended use is not permitted by statutory regulation or exceeds the permitted use, you will need to obtain permission directly from the copyright holder. To view a copy of this licence, visit http://creativecommons. org/licenses/by/4.0/.

\section{References}

1. Zhai W, Zhao C (2016) Frontiers and challenges of sciences and technologies in modern railway engineering. J Southwest Jiaotong Univ 51(2):209-226 (in Chinese)

2. Cai C, He Q, Zhu S et al (2019) Dynamic interaction of suspension-type monorail vehicle and bridge: Numerical simulation and experiment. Mech Syst Signal Process 118:388-407

3. Kouroussis G, Vogiatzis KE, Connolly DP (2018) Assessment of railway ground vibration in urban area using in-situ transfer mobilities and simulated vehicle-track interaction. Int J Rail Transp 6(2):113-130

4. Yasuda Y, Fujino M, Tanaka M, et al (2004) The first HSST maglev commercial train in Japan. In: Proceedings of MAGLEV 2004, October 26-28, Shanghai, 1: 76-85

5. Lim J, Jeong JH, Kim CH et al (2017) Analysis and experimental evaluation of normal force of linear induction motor for maglev vehicle. IEEE Trans Magn 53(11):1-4

6. Sun Y, Xu J, Qiang H et al (2019) Adaptive neural-fuzzy robust position control scheme for maglev train systems with experimental verification. IEEE Trans Industr Electron 66(11):8589-8599

7. Xu F, Luo S, Deng Z (2019) Study on key technologies and whole speed range application of maglev rail transport. J China Railw Soc 41(3):40-49 (in Chinese) 
8. Ma W-H, Luo S-H, Zhang M et al (2021) Research review on medium and low speed maglev vehicle. J Traffic Transp Eng 21(1):199-216 (in Chinese)

9. Zhou D, Yu P, Wang L et al (2017) An adaptive vibration control method to suppress the vibration of the maglev train caused by track irregularities. J Sound Vib 408:331-350

10. Zhai W, Xia H, Cai C et al (2013) High-speed train-track-bridge dynamic interactions-part I: theoretical model and numerical simulation. Int J Rail Transp 1(1-2):3-24

11. Zhai W, Wang S, Zhang $\mathrm{N}$ et al (2013) High-speed train-trackbridge dynamic interactions-part II: experimental validation and engineering application. Int J Rail Transp 1(1-2):25-41

12. Zhai W, Han Z, Chen $Z$ et al (2019) Train-track-bridge dynamic interaction: a state-of-the-art review. Veh Syst Dyn 57(7):984-1027

13. Zhai W (2020) Vehicle-track coupled dynamics: theory and applications. Springer, Singapore

14. Gou H, Liu C, Zhou W et al (2020) Dynamic responses of a highspeed train passing a deformed bridge using a vehicle-trackbridge coupled model. Proc Inst Mech Eng Part F J Rail Rapid Transit 235(4):463-477

15. Yaghoubi H, Ziari H (2011) Development of a maglev vehicle/ guideway system interaction model and comparison of the guideway structural analysis with railway bridge structures. J Transp Eng Part A Syst 137(2):140-154

16. Han H, Yim BH, Lee NJ et al (2009) Effects of the guideway's vibrational characteristics on the dynamics of a maglev vehicle. Veh Syst Dyn 47(3):309-324

17. Kim K-J, Han J-B, Han H-S et al (2015) Coupled vibration analysis of maglev vehicle-guideway while standing still or moving at low speeds. Veh Syst Dyn 53(4):587-601

18. Lee JS, Kwon SD, Kim MY et al (2009) A Parametric study on the dynamics of urban transit maglev vehicle running on flexible guideway bridges. J Sound Vib 328(3):301-317

19. Kwon SD, Lee JS, Moon JW et al (2008) Dynamic interaction analysis of urban transit maglev vehicle and guideway suspension bridge subjected to gusty wind. Eng Struct 30(12):3445-3456

20. Wang D, Li X, Wu Z (2020) Dynamic performance of the LMS maglev train-track-bridge system under uneven settlement for two typical bridges. Int J Struct Stab Dyn 21(1):2150006

21. Yau JD (2009) Response of a maglev vehicle moving on a series of guideways with differential settlement. J Sound Vib 324(3):816-831

22. Yau JD (2013) Wave passage effects on the seismic response of a maglev vehicle moving on multi-span guideway. Latin Am J Solids Struct 10(5):981-1000

23. Han JB, Han HS, Kim SS et al (2016) Design and validation of a slender guideway for maglev vehicle by simulation and experiment. Veh Syst Dyn 54(3):370-385

24. Chen X, Ma W, Luo S (2020) Study on stability and bifurcation of electromagnet-track beam coupling system for EMS maglev vehicle. Nonlinear Dyn 101(3):2181-2193

25. Wang D, Li X, Wang Y et al (2020) Dynamic interaction of the low-to-medium speed maglev train and bridges with different deflection ratios: experimental and numerical analyses. Adv Struct Eng 23(11):2399-2413
26. Wang D, Li X, Liang L et al (2019) Influence of the track structure on the vertical dynamic interaction analysis of the lowto-medium-speed maglev train-bridge system. Adv Struct Eng 22(14):2937-2950

27. Han JB, Han HS, Lee JM et al (2018) Dynamic modeling and simulation of EMS maglev vehicle to evaluate the levitation stability and operational safety over an elastic segmented switch track. J Mech Sci Technol 32(7):2987-2998

28. Li X, Wang D, Liu D et al (2018) Dynamic analysis of the interactions between a low-to-medium-speed maglev train and a bridge: field test results of two typical bridges. Proc Inst Mech Eng Part F J Rail Rapid Transit 232(7):2039-2059

29. Li M, Luo S, Ma W et al (2021) Experimental study on dynamic performance of medium and low speed maglev train-trackbridge system. Int J Rail Transp 9(3):232-255

30. Sun Y, Qiang H, Xu J et al (2020) Internet of Things-based online condition monitor and improved adaptive fuzzy control for a medium-low-speed maglev train system. IEEE Trans Industr Inf 16(4):2629-2639

31. Chen C, Xu J, Lin G et al (2020) Model identification and nonlinear adaptive control of suspension system of high-speed maglev train. Veh Syst Dyn. https://doi.org/10.1080/00423114. 2020.1838564

32. Kong E, Song J, Kang B et al (2011) Dynamic response and robust control of coupled maglev vehicle and guideway system. J Sound Vib 330(25):6237-6253

33. Li S, Zhang K (2015) Self-excited vibration of single-magnet suspension system: stability analysis and inhibition. J Southwest Jiaotong Univ 50(3):410-416 (in Chinese)

34. Wang H-P, Li J, Zhang K (2007) Stability and hopf bifurcation of the maglev system with delayed speed feedback control. Acta Autom Sinica 33(8):829-834

35. Zhang L, Huang L, Zhang Z (2009) Stability and hopf bifurcation of the maglev system with delayed position and speed feedback control. Nonlinear Dyn 57(1):197-207

36. Liu DL (2017) Experimental study on dynamic load test of medium and low-speed maglev switch system. Urban Mass Transit 20(5):89-92 (in Chinese)

37. Zhang Y, Zhao C, Zhou W et al (2020) Natural vibration characteristics of the active turnout girder with different boundary conditions for low-medium speed maglev transit. Railw Stand Des 64(4):22-27 (in Chinese)

38. Luo HJ, Wu ZH, Tong L-S et al (2018) Research on vehicle and turnout coupling vibration for mid-low speed maglev. Electr Locomot Mass Transit Veh 41(1):5-8 (in Chinese)

39. Chai X-P, Wang Z-X, Wang B et al (2017) Study of TLMD vibration mitigation technique for switch beams in maglev project. World Bridg 45(2):60-65 (in Chinese)

40. Zeng G, Yuan Y, Ji W et al (2016) Design and debugging of guideway switch for medium and low-speed maglev in Changsha city. Urban Mass Transit 19(5):44-48 (in Chinese)

41. Zhao C, Zhai W (2002) Maglev vehicle/guideway vertical random response and ride quality. Veh Syst Dyn 38(3):185-210 